Research article

\title{
An efficient genetic algorithm for structural RNA pairwise alignment and its application to non-coding RNA discovery in yeast
} Akito Taneda

\author{
Address: Graduate School of Science and Technology, Hirosaki University, 3 Bunkyo-cho, Hirosaki, Japan \\ Email: Akito Taneda - taneda@eit.hirosaki-u.ac.jp
}

Published: 5 December 2008

BMC Bioinformatics 2008, 9:521 doi:10.1/86/147|-2105-9-521
Received: 2 September 2008

Accepted: 5 December 2008

This article is available from: http://www.biomedcentral.com/l47|-2105/9/52I

(c) 2008 Taneda; licensee BioMed Central Ltd.

This is an Open Access article distributed under the terms of the Creative Commons Attribution License (http://creativecommons.org/licenses/by/2.0), which permits unrestricted use, distribution, and reproduction in any medium, provided the original work is properly cited.

\begin{abstract}
Background: Aligning RNA sequences with low sequence identity has been a challenging problem since such a computation essentially needs an algorithm with high complexities for taking structural conservation into account. Although many sophisticated algorithms for the purpose have been proposed to date, further improvement in efficiency is necessary to accelerate its large-scale applications including non-coding RNA (ncRNA) discovery.

Results: We developed a new genetic algorithm, Cofolga2, for simultaneously computing pairwise RNA sequence alignment and consensus folding, and benchmarked it using BRAliBase 2.I. The benchmark results showed that our new algorithm is accurate and efficient in both time and memory usage. Then, combining with the originally trained SVM, we applied the new algorithm to novel ncRNA discovery where we compared $S$. cerevisiae genome with six related genomes in a pairwise manner. By focusing our search to the relatively short regions (50 bp to 2,000 bp) sandwiched by conserved sequences, we successfully predict $7 / 4$ intergenic and I,3II sense or antisense ncRNA candidates, which were found in the pairwise alignments with stable consensus secondary structure and low sequence identity $(\leq 50 \%)$. By comparing with the previous predictions, we found that $>92 \%$ of the candidates is novel candidates. The estimated rate of false positives in the predicted candidates is $51 \%$. Twenty-five percent of the intergenic candidates has supports for expression in cell, i.e. their genomic positions overlap those of the experimentally determined transcripts in literature. By manual inspection of the results, moreover, we obtained four multiple alignments with low sequence identity which reveal consensus structures shared by three species/sequences.
\end{abstract}

Conclusion: The present method gives an efficient tool complementary to sequence-alignmentbased ncRNA finders.

\section{Background}

The RNA worlds in both experimental and computational fields have recently grown rapidly, and non-coding RNAs (ncRNAs) have increased their importance in life sciences. One of the most important breakthrough from the experimental side is the high-throughput experiments which have unveiled the existence of many non-protein coding transcripts in various species [1,2]. While function-known ncRNAs, which often harbor family-specific conserved secondary structure, such as tRNAs and miRNAs have been intensively studied in detail, no functional annotation has been assigned to a number of known non-protein coding transcripts yet. Since experimental assessment whether all known non-protein coding transcripts are 
functional or not is quite time-consuming, computational screening for finding the ncRNAs with conserved secondary structure is an important step for determining not only expressed but also functional transcripts. Computational comparative genomics is a powerful approach to identify ncRNA candidates with conserved secondary structure from genomic sequences. To date, sequence-alignmentbased ncRNA finders such as RNAz [3], QRNA [4] and EvoFold [5] have been successfully applied to ncRNA discoveries from various complete genomes [6-10]. While these methods are so efficient that they can be applied to genome-scale analysis, sequence-alignment-based methods need a pre-computed alignment as an input data. In other words, they implicitly assume that an adequately accurate RNA sequence alignment can be obtained by using pure sequence alignment method (e.g. ClustalW) which does not explicitly consider conserved secondary structure. Although this assumption is acceptable for the RNA sequences with relatively high sequence identity, sequence-alignment-based methods can fail to indentify the ncRNAs with low sequence identity; this is because conserved secondary structure should be taken into account to accurately align structured RNA sequences which are poorly conserved at sequence level.

Finding related structured RNA sequences with low sequence identity from genomic sequences is more challenging compared to the case of high sequence identity. This is mainly due to the high computational complexities of structural RNA sequence alignment algorithms which explicitly take secondary structure into account (in the present paper, the term "structural RNA sequence alignment" is used to indicate "simultaneously determining RNA sequence alignment and conserved secondary structure without pre-defined secondary structure annotation"). For example, the computational complexities of Sankoff's algorithm which is the most basic algorithm for structural RNA sequence alignment are $O\left(N^{3 M}\right)$ in time and $O\left(N^{2 M}\right)$ in space, where $N$ and $M$ are the length and the number of RNA sequences to be aligned, respectively [11]. Even when one performs pairwise alignment, Sankoff's algorithm needs $O\left(N^{6}\right)$ in time and $O\left(N^{4}\right)$ in space. To improve the computational speed and memory usage of structural RNA sequence alignment, various variations of Sankoff's algorithm have been intensively studied [12-21].

So far, Dynalign [13] and Foldalign [14] which are variations of Sankoff's algorithm, have been applied to the pairwise comparative genomics for novel ncRNA discoveries[22,23]. Indeed these 'structure-based' ncRNA finders have successfully predicted a number of ncRNA candidates with low sequence identities, these calculations needed long computational times and large computational resources. Although these programs have been updated recently and the latest versions are faster compared to their older versions, it is still time consuming to apply these programs to genome-scale applications. Recently an efficient structural RNA sequence alignment algorithm, LocARNA, has been proposed[15]. To our knowledge, however, there is no report related to the ncRNA discovery by using LocARNA. Since genomic scans by previous structural RNA sequence alignment methods are time consuming and need large computational resources in general, further development of efficient and accurate structural RNA sequence alignment algorithm is important to accelerate the genome-scale prediction of the ncRNAs with low sequence identities. Recently, CMfinder, which is structural RNA sequence alignment algorithm not for pairwise but for multiple RNA sequence alignment, has successfully predicted a number of novel structured RNA motifs from the ENCODE regions with low sequence identities [24].

In the present paper, we propose an improved genetic algorithm (GA), Cofolga2, for structural RNA pairwise alignment which uses the base pairing probabilities (BPPs) by RNAfold[25] to evaluate the structural term of the objective function instead of directly using the free energy parameters as its version 1 does [21]. Since the present algorithm is efficient in both time and memory usage, we applied the algorithm to the pairwise comparisons between eukaryotic complete genomes to search for novel ncRNA candidates from low sequence identity regions. The rest of the present paper is organized as follows. First we describe the present structural RNA sequence alignment algorithm and a strategy for our comparative genomics in the 'Methods' section. In the next section, we show the results of the benchmark and the comparison between the present algorithm and previous ones, discussing the performance of our alignment algorithm. Finally, we present the detail of the ncRNA candidates obtained by the pairwise genome comparisons between $S$. cerevisiae and other six fungi.

\section{Methods}

In Cofolga2 algorithm, we employ a GA to search for the optimal solution of structural RNA pairwise alignment. Cofolga2 is an updated version of the previously proposed GA [21] which performs structural RNA pairwise alignment based on minimization of free energy and the GA frameworks proposed in RAGA [26] (in the present paper, we call the previous version as Cofolga1). Cofolga 2 runs much faster compared to Cofolga1; this is mainly due to the improvement in the formulation of objective function and introduction of a new technique for random alignment generation. In standard GA, various GA operators (crossovers and mutations) are iteratively applied to a population of individuals (solutions) to search for the optimal solution with the highest value of a given objec- 
tive function (OF) [27]. In the Cofolga algorithms, an individual of GA is represented by a pairwise alignment. This is because structural RNA sequence alignment problem can be decomposed into sequence alignment and alignment folding, and the optimal alignment folding is uniquely defined for a given alignment. As a result, the conformational space to be explored in the present structural RNA pairwise alignment is reduced to that of nonstructural pairwise sequence alignment.

The OF of Cofolga2 is represented by the following formula:

$$
f=s+w P,
$$

where $s$ is a sequence alignment score, $P$ is a term for consensus secondary structure; $w$ is a parameter for controlling the weights of $s$ and $P$.

For a given pairwise alignment of RNA sequence $\mathrm{A}$ and $\mathrm{B}$, the $P$ in Equation 1 is evaluated as follows.

First an averaged BPP matrix $B$ is constructed:

$$
b_{i j}= \begin{cases}\left(p_{k_{i} l_{j}}^{\mathrm{A}}+p_{m_{i} n_{j}}^{\mathrm{B}}\right) / 2 & p_{k_{i} l_{j}}^{\mathrm{A}} \neq 0 \text { and } p_{m_{i} n_{j}}^{\mathrm{B}} \neq 0 \\ 0 & \text { otherwise. }\end{cases}
$$

In Equation $2, b_{i j}$ is the matrix element of $\boldsymbol{B}$, where $i$ and $j$ indicate the column positions in the pairwise alignment; $k_{i}$ and $l_{j}\left(m_{i}\right.$ and $\left.n_{j}\right)$ are the nucleotide positions in sequence A (sequence B) corresponding to column position $i$ and $j$ in the pairwise alignment, respectively. The BPPs of single sequence $\mathrm{A}$ and $\mathrm{B}, p_{k l}^{\mathrm{A}}$ and $p_{m n}^{\mathrm{B}}$, are computed by RNAfold [25]. Secondly, the $P$ is evaluated by taking a summation of the elements in matrix $B$ :

$$
P=\sum_{i<j} b_{i j}
$$

It is noteworthy that Equation 3 can be applied to any type of pseudoknotted structure without modification. This means that once the BPP matrixes taking pseudoknots into account are given, Cofolga2 algorithm can perform structural RNA sequence alignment of pseudoknotted RNAs without an increase of computational costs compared to the case of non-pseudoknotted RNAs.

The flowchart of Cofolga2 algorithm is shown in Figure 1. In accordance with the standard GA, first, initialization is done to randomly generate an initial population, and then evaluation and reproduction procedures are itera- tively performed to update the population. This iteration stops when the number of iterations reaches a userdefined maximum number or when no improvement has been observed for a user-defined number of iterations.

As mentioned above, Cofolga2 was developed based on Cofolga1. In the following subsections, we will focus on explaining the detail of algorithms newly introduced for Cofolga2. Algorithmically common parts between the two versions will be briefly explained.

\section{Initialization}

An initial population of solutions is generated by adding a randomly generated pairwise alignment to the population one by one until the number of individuals reaches a user-defined population size. The random pairwise alignments are computed by using weighted stochastic backtracking (for detail, see next subsection). In weighted stochastic backtracking, the randomness of the alignment can be controlled with a 'noise' parameter where larger noise gives more randomized alignment; based on our experience, we used noise $=0.1-0.4$ to obtain random alignments.

In addition to the random alignments, a non-random alignment taking structure information into account (computed by weighted stochastic backtracking with a very small noise such as noise $=1.0 \times 10^{-4}$ ) and a nonstructural Needleman-Wunsch alignment [28] can also be included in the initial population through a command line option (a '-nrd' option). When invoked with the '-nrd' option, Cofolga 2 works as a refinement program which improves the two non-random alignments. Inclusion of the non-structural alignment improves the quality of the alignments with a relatively high sequence identity. In the default setting of Cofolga2, duplicated individuals in one population are not allowed throughout the run.

\section{Weighted stochastic backtracking}

In the initialization step of the alignment algorithms utilizing GA such as SAGA [29] and RAGA [26], it is necessary to generate a number of random alignments. For example, in RAGA algorithm, random pairwise alignments are computed by using a Dynamic Programming with Added Noise (DPAN) in which random alignments are obtained by adding small random noises to each DP matrix elements [30]. Since DPAN constructs a DP matrix in accordance with non-structural Needleman-Wunsch algorithm, structural information is completely lacked in such a calculation. To obtain a better initial guess for the structural RNA sequence alignment, structural information should be taken into account.

To generate random pairwise alignments which reflect structural information, we developed weighted stochastic 


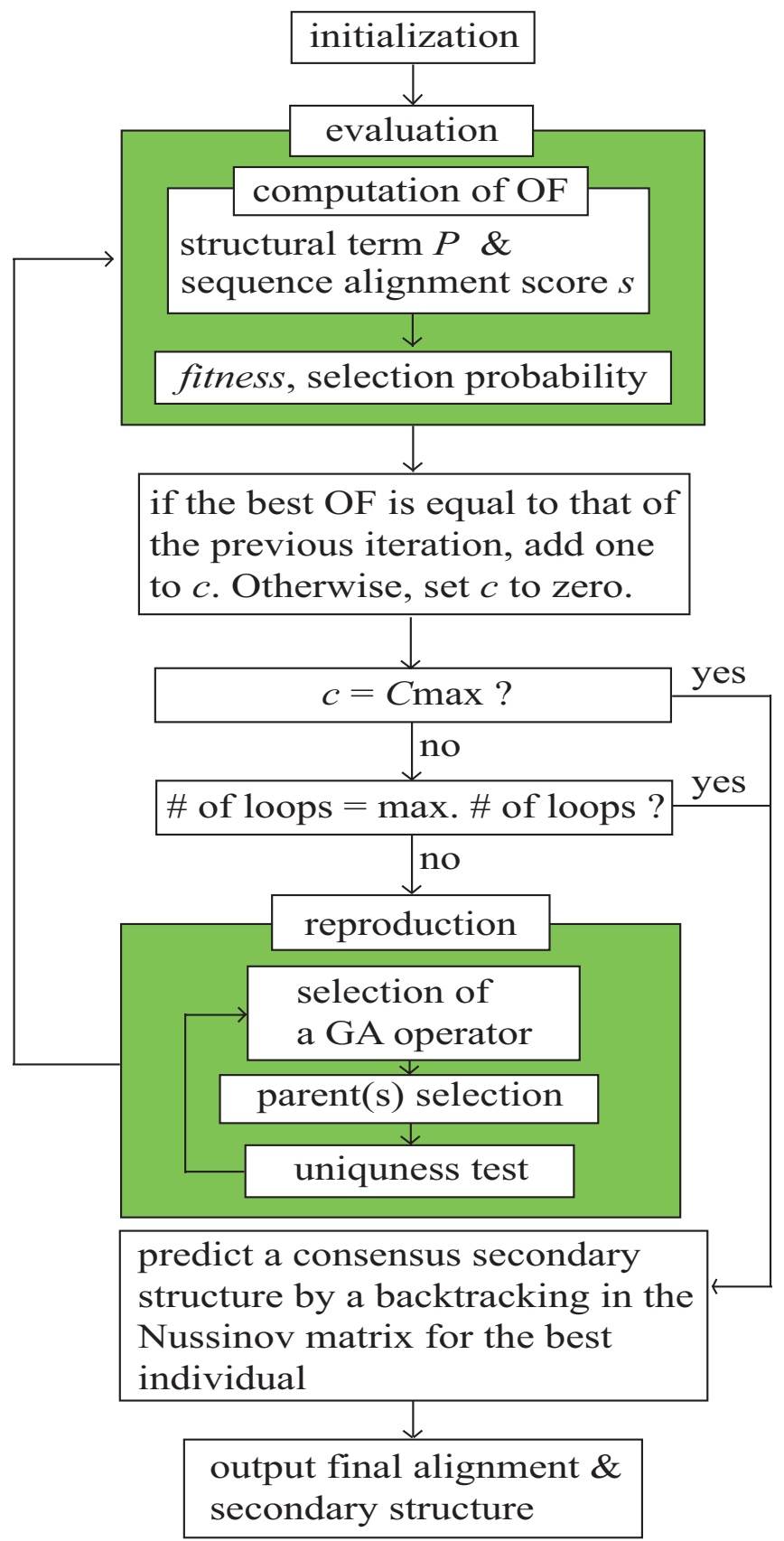

Figure I

Schematic flowchart of Cofolga2 algorithm. Cofolga2 algorithm is composed of three GA steps (initialization, evaluation, and reproduction) and a postprocessing step. In initialization, a population of individuals is randomly generated by weighted stochastic backtracking. In evaluation step, the objective function of each individual is evaluated and then fitness and selection probability are assigned to each individual. In reproduction, half of the population is replaced by new individuals to update the population. The iteration between evaluation and reproduction stops when one of the following conditions is satisfied: the best $\mathrm{OF}$ is not updated continuous $C_{\max }$ times, or the number of iteration reaches a pre-defined maximum iteration number. The $C_{\max }$ and the maximum iteration number are parameters given by user. A consensus secondary structure prediction for the optimal alignment is performed as a postprocessing, where the Nussinov matrix constructed by averaged base pairing probabilities is backtracked. 
backtracking. In this algorithm, first, we construct the DP matrix for a pairwise alignment according to StrAl algorithm [31]. StrAl algorithm is an efficient structural alignment algorithm, and it was derived from an affine gap version of Needleman-Wunsch algorithm [28]. An essential difference between StrAl and the Needleman-Wunsch algorithm is their similarity scoring scheme. In StrAl algorithm, the following similarity score $s_{i j}$ is used when constructing the DP matrix for pairwise alignment instead of the nucleotide substitution matrix $d\left(A_{i}, B_{j}\right)$ alone:

$$
\begin{gathered}
s_{i j}=\alpha\left(\sqrt{\kappa_{i}^{\mathrm{A}} \kappa_{j}^{\mathrm{B}}}+\sqrt{\lambda_{i}^{\mathrm{A}} \lambda_{j}^{\mathrm{B}}}\right)+d\left(A_{i}, B_{j}\right) \sqrt{\mu_{i}^{\mathrm{A}} \mu_{j}^{\mathrm{B}}} \\
\kappa_{i}^{\mathrm{X}}=\sum_{k<i} p_{i k^{\prime}}^{\mathrm{X}} \lambda_{i}^{\mathrm{X}}=\sum_{k>i} p_{i k^{\mathrm{X}}}^{\mathrm{X}} \\
\mu_{i}^{\mathrm{X}}=1-\left(\kappa_{i}^{\mathrm{X}}+\lambda_{i}^{\mathrm{X}}\right)
\end{gathered}
$$

where $s_{i j}$ indicates the similarity score between position $i$ of sequence $\mathrm{A}$ and position $j$ of sequence $\mathrm{B}$, and $\alpha$ is the ratio of structure over sequence similarity. Nucleotide substitution matrix element $d\left(A_{i}, B_{j}\right)$ is the substitution score between the $i$ th nucleotide of sequence $A$ and the $j$ th nucleotide of sequence $B$. In the present study, we used RIBOSUM85-60 [32] for $d\left(A_{i}, B_{j}\right)$ and $\alpha=0.9$ which was taken from the StrAl paper [31]. Base paring probability vectors $\kappa_{i}^{\mathrm{X}}, \lambda_{i}^{\mathrm{X}}$, and $\mu_{i}^{\mathrm{X}}$ are the probabilities defined for the position $i$ of sequence $\mathrm{X}$ (= A or $\mathrm{B}$ ) which represent probabilities of being paired upstream, paired downstream, and unpaired, respectively. The affine gap penalties which we used for weighted stochastic backtracking are also taken from the StrAl paper [31].

After the construction of the DP matrix, we backtrack the DP matrix in accordance with a roulette wheel selection. Roulette wheel selection is a selection method frequently used in GAs, in which one of all choices is randomly chosen in accordance with the probability proportional to the size of a virtual 'slot' assigned to the choice.

The size of the slots is determined by the following scaling function:

$$
\text { slot }_{i}=\frac{\text { noise }}{h_{\max }-h_{i}+\text { noise }},
$$

where index $i$ corresponds to a backtracking path at a node of the DP matrix ( $i=1,2,3$ for pairwise alignment), $h_{i}$ is the score difference between the current node and the neighboring node for path $i$, and $h_{\max }$ is the largest $h_{i}$ among $h_{1}, h_{2}$, and $h_{3}$. Larger noise parameter noise generates a more randomized alignment. A backtracking path is chosen in accordance with backtracking probability $\pi_{i}$ which is defined as follows:

$$
\pi_{i}=\operatorname{slot}_{i} / \sum_{i=1,2,3} \operatorname{slot}_{i}
$$

While backtracking, a real random number ranging from 0 to 1 is generated at each node and used to select a next path to be backtracked.

In Figure 2, the curves drawn by the scaling function are plotted. As can be seen from the figure, higher noise increases the probability to choose low scoring paths, while noise $\rightarrow 0$ means the optimal alignment. Thus, the randomness of weighted stochastic backtracking is controllable through the single parameter noise. It is noteworthy that the principles of weighted stochastic backtracking can easily be applied to any type of DP algorithm, e.g. those of Nussinov's algorithm [33] and Sankoff's algorithm [11].

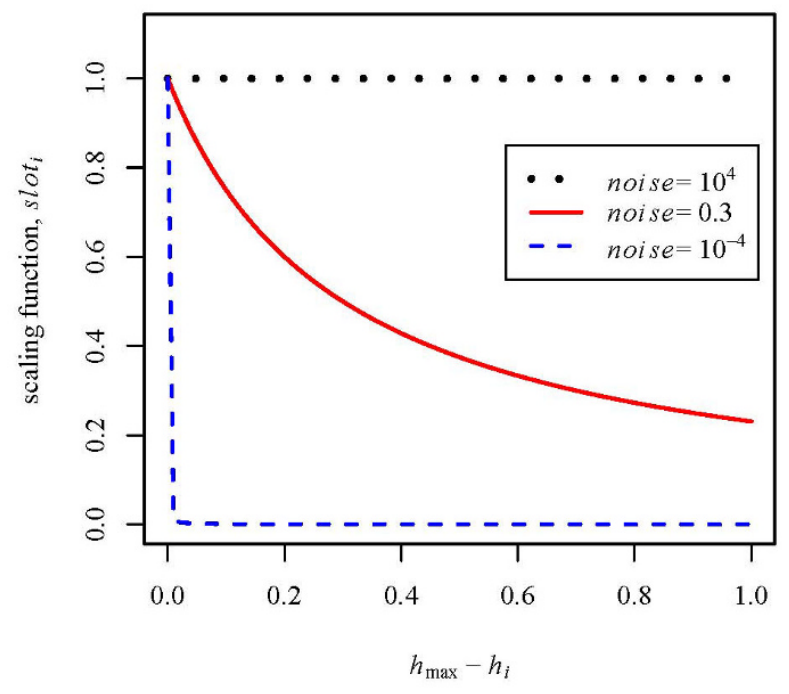

\section{Figure 2}

Scaling function for weighted stochastic backtracking. The curves by the proposed scaling function, slot $_{i}=$ noise/ $(x+$ noise $)$, are plotted, where $x$ is the score difference between the optimal and the other backtracking path (i.e. $x=$ $h_{\max }-h_{i}$ in Equation 7). In the figure, dotted line, solid line, and dashed line indicate the curves for noise $=10^{4}$, noise $=$ 0.3 , and noise $=10^{-4}$, respectively (noise $=10^{4}$ and noise $=10^{-4}$ are drawn as examples of two extreme cases). For any noise $>0$, the optimal path $(x=0)$ has slot $\max _{\max }=1$, while damped slot sizes $(<1)$ are assigned to the non-optimal DP paths $(x>$ 0 ). 


\section{Evaluation}

The OF, $f$ in Equation 1, of each individual is evaluated in this step, where the alignment score $s$ is calculated by using the RIBOSUM85-60 [32]. Opening and elongation gap penalties are left as free parameters. After the evaluation of the OF, the fitness of each individual is computed from the OF as fitness = OF - (the lowest $O F$ in the population), and then a selection probability proportional to the fitness is calculated for each individual. The selection probability is used in reproduction step as the size of virtual slots for the roulette-wheel selection of parent individuals.

\section{Reproduction}

In reproduction step, half of the population with the lowest OFs is replaced by new child individuals. The child individuals are generated by applying GA operators to the parent individuals randomly selected from the population. We use a modified set of the GA operators taken from Cofolga1[21], which is comprised of two crossovers (random and greedy two-point crossovers) and three mutations (random and greedy gap-block shuffling operators, local re-alignment with weighted stochastic backtracking). Each GA operator is invoked with an equal probability and applied to one or two randomly selected parent individual(s); the crossovers need two parents, while the other operators are applied to a single parent. Selection of parent(s) is performed by roulette-wheel selection where the selection probability of each individual is used as the size of the slots. The GA operators are schematically illustrated in Figure 3. Crossover operators construct a new alignment by concatenating 'alignment blocks' taken from two parent individuals. Gap-block shuffling operator 'shuffles' a gap block (a block of continuous gaps) by a random shift size in a random direction. The maximum size of the gap shift is defined by shift size parameter max_shift.

In 'local re-alignment with weighted stochastic backtracking', a randomly selected small region of the alignment is re-aligned using weighted stochastic backtracking. The region to be re-aligned is selected by the following gapsensitive procedure. First, we initialize $\rho[i]=1$ for all $i$, where $i$ indicates the column position of the alignment. Secondly, we scan the alignment with a sliding window of $W$ columns. While scanning the alignment, we count the number of gaps in each window and add the number to the $\rho[i]$ whose $i$ is the center of the sliding window. Thirdly, a column position $k$ is randomly selected in accordance with the probability proportional to $\rho[i]$. Finally, we define the region to be re-aligned around the $k$. The width of the region is randomly determined between $l_{\min }$ and $l_{\max }$. When we meet a trivial case (i.e. when one of the alignment rows included in the region has no nucleotides), this operator is rejected and a next (a) two point crossover

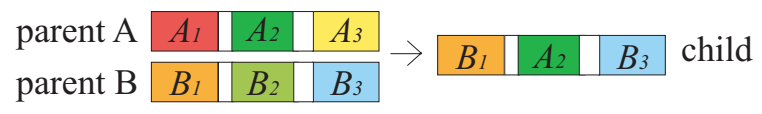

(b) gap-block shuffling

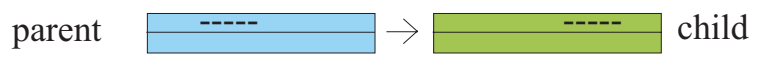

(c) local re-alignment

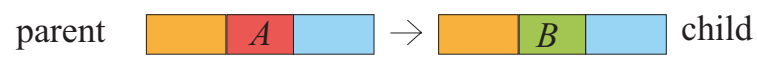

\section{Figure 3}

Schematic illustration of GA operators. Different colors of the blocks indicate different alignment blocks. White blocks indicate equivalent blocks between two pairwise alignments. (a) Two-point crossover creates a child alignment by concatenating at most three alignment blocks separated by equivalent blocks. When the number of the separated blocks is larger than three, the smallest blocks are merged to neighboring blocks in an iterative manner. (b) Gapblock shuffling shifts a randomly selected continuous gap in a random direction. (c) Local re-alignment re-aligns a randomly selected small alignment region. In this example, the central region of the alignment is modified, while the flanking regions are not changed.

GA operator is randomly invoked. In this procedure, the $W, l_{\min }$ and $l_{\max }$ are the parameters to be given by user.

In general, GA has several free parameters such as population size and iteration number which have to be given before execution. To reduce the number of such free parameters, we introduced unification parameter $L$. This $L$ defines several parameters for the GA operators simulta-

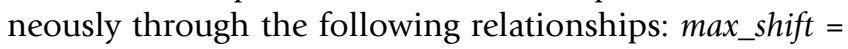
$l_{\max }=L$ and $l_{\min }=(W-1) / 2=\& \# \times 230 A ; L / 2 \& \# \times 230 B ;$. Hence once $L$ is given, max_shift, $l_{\max ^{\prime}} l_{\min ^{\prime}}$ and $W$ are determined and only $L$ is left as a free parameter. Unification parameter $L$ controls the degree of modification, i.e. larger $L$ leads to a wider search in the conformational space by the mutation operators. It is noted that too large $L$ can cause a slow convergence of the GA.

In the nomenclature of the GA operators, a 'greedy' means that the operator increases the OF of the child individual compared to that of its parents. Cofolga2 uses 'greedy' operators while Cofolga1 uses 'semi-greedy' operators. The 'greedy' operators of Cofolga 2 reject the child individuals which do not satisfy (the OF of the child individual) $>$ (the OF of the parent individual(s)) while the 'semigreedy' operators of Cofolga1 does not. Cofolga2 does not utilize 'anchor point for mutation operators' which is used in Cofolga 1 to avoid gap insertion into highly conserved regions of an alignment (subsection 2.3.2 in [21]). 
Local Cofolga operator (ibid., subsection 2.3.6) is also not used in Cofolga2.

\section{Consensus structure prediction by a postprocessing}

Cofolga2 predicts the consensus secondary structure for the final alignment by backtracking in the averaged BPP matrix as a postprocessing of the GA procedures. To more accurately predict a consensus secondary structure based on the alignment computed by Cofolga2, it is better to use an alignment folding program such as RNAalifold [34] or Pfold [35] as a postprocessing.

\section{Measures for assessing alignment quality}

The quality of pairwise alignments was assessed with structure conservation index (SCI) and sum-of-pairs score (SPS). SCI and SPS were evaluated by RNAz [3] and bali_score.c [36], respectively.

\section{Determination of the free parameters}

In addition to the population size and maximum iteration number of the GA, Cofolga2 has six free parameters: $C_{\max }$ noise, $w$, gap opening and elongation penalties for $s$, and $L$ . We optimized these six parameters with fourteen pairwise alignments taken from the $\mathrm{k} 2$ dataset of BRAliBase 2.1 [37]. The training RNA sequences are tRNAs, 5S rRNAs, and SRP RNAs with high or low sequence identities and high or low SCIs. For tRNA, alignments with a moderate sequence identity were also used. The file names of the RNA sequences are listed in Additional File 1. The parameter space to be explored was represented by a coarse grid and the parameter set corresponding to the grid point which scored the highest (mean SPS) $\times$ (mean SCI ) was adopted as the optimal parameter set $\left(C_{\max }=50\right.$, noise $=0.3, w=50$, gap opening $=30$, gap elongation $=4$, and $L=50$ ). Throughout this optimization, we used a population size and maximum iteration number fixed to relatively large values, 150 and 150, respectively. The results presented at the Results and discussion section were obtained with this optimal parameter set. The popu- lation size and maximum iteration number are left as free parameters.

\section{Benchmark and comparison of alignment quality}

We performed an alignment quality benchmark using BRAliBase 2.1 [37] from which RNA sequence pairs and their reference alignments were taken. In addition, we performed performance comparison with other structural and non-structural sequence alignment programs using the benchmark. In the performance comparison, we compared Cofolga 2 with five structural and three non-structural alignment methods. The programs and command line options are summarized in Table 1 . To perform the comparisons on an equal footing, global alignment mode was used for local alignment programs, Foldalign and LOCARNA.

\section{Benchmark for the sequence pairs with low identities}

In addition to the BRaliBase 2.1 benchmark, we have performed a benchmark with the sequences which have identities $\leq 40 \%$ and lengths of 100 to $150 \mathrm{nt}$. The sequences were extracted from the internal transcribed spacer 2 (ITS2) database [38], where the sequences and annotated structures of Stramenopiles and "the original 5,000 sequences and structures" (ITS2.html, [39]) were used. Sequence identities were measured after aligning two ITS2 sequences using MAFFT (see Table 1). We have performed non-redundant processing with a cutoff of $90 \% \mathrm{id}$. As a result, we obtained twenty-five ITS2 sequence pairs (the ITS2 dataset can be browsed at the Cofolga 2 website [40]); the average sequence identity of the dataset is 33\%. This benchmark was performed for Cofolga2, Foldalign 2.1.0, and LocaRNA. Since annotated secondary structures are given in the ITS2 database and reference alignments are not provided, the prediction accuracy for this benchmark was measured based on how correctly annotated (reference) base pairs are predicted. The correctness of the predicted base pairs was assessed with the approximated

Table I: Alignment programs used for performance comparisons

\begin{tabular}{lrlrl}
\hline program & version & command & structural? & reference \\
\hline Foldalign & 2.1 .0 & foldalign-global seq_filename & yes & {$[48]$} \\
LocARNA & 0.99 & mlocarna-p-sequ-local =0 seq_filename & yes & {$[15]$} \\
Dynalign & $*$ & cat opt_file | dynalign & yes & yes \\
LaRA & 1.3 .1 & lara-i seq_filename & yes & {$[5]$} \\
StrAl & 0.5 .2 & stral seq_filename & no & {$[56]$} \\
MAFFT & 6.240 & ginsi seq_filename & no & {$[57]$} \\
ClustalW & 1.83 & clustalw seq_filename-outfile = out_filename & {$[58]$} &
\end{tabular}

In this table, 'program' and 'version' columns indicate program names and their versions, respectively. In 'command' column, executable file names and options we used are listed. In 'structural?' column, 'yes' indicates structural RNA sequence alignment program, and 'no' represents nonstructural sequence alignment program. RNAz I.0 with a default setting was applied to the alignments provided by MAFFT and ClustalW. $(*)$ Dynalign version 4.5 (with $M=-99$ ) was used for the alignment quality benchmark, and Dynalign version 4.3 (with $M=8$ ) was used for the ncRNA prediction benchmark, where we used the values recommended by Dynalign for the other parameters except for the maximum number of structures $=1$. The parameters and file names were written in opt_file and used to run Dynalign. 
Matthews correlation coefficient (Equation 5 in [41]), CC, proposed by Gorodkin et al..

SVM classification between true ncRNAs and shuffled data To predict ncRNAs on the basis of the pairwise alignment computed by Cofolga2, we trained SVM by using a SVM package software, LIVSVM (version 2.84) [42]. The elements of the feature vector for the SVM are as follows: OF, alignment length, and $\mathrm{A}, \mathrm{C}$, and $\mathrm{U}$ frequencies of the two sequences. These quantities except for the OF were calculated after eliminating all gapped columns of the alignment. The alignments $<50 \mathrm{nt}$ were removed from the input before SVM processing. This format of the feature vector is taken from the paper describing the ncRNA finding by Dynalign [43]. We use a default kernel (radial basis function kernel), and the prediction result of the SVM is outputted as a classification probability. To construct positive training and test datasets, we extracted 5,010 pairwise alignments from the $\mathrm{k} 2$ dataset of BRAliBase2.1 [37]. The sequence identity of this dataset ranges from $16 \%$ to $75 \%$ and the dataset comprises thirtytwo RNA families. This original dataset was divided into two sub-datasets in a ratio of 1:2 (1,670 alignments for training, 3,340 alignments for test). Negative data were generated by removing all gapped columns of the positive alignments and shuffling the gap-free alignments. Two negative alignments were generated for each positive alignment, consequently we obtained 3,340 negatives for training and 6,680 negatives for test. The shuffling was performed by shufflealn.pl [34] with a '-m complete' option. After the training, we obtained a test accuracy of $87.7 \%$.

\section{Visualization of ncRNA prediction performance}

When the performance of prediction methods depends on their own cutoff value, comparison of the methods becomes not straight forward, since varying the cutoff value leads to a simultaneous change of sensitivity and specificity (i.e. there is a tradeoff between sensitivity and specificity).

In the present study, we used receiver operating characteristic (ROC) curve for visualizing the tradeoff between sensitivity and specificity for a range of cutoff value. The ROC curve has been used by Uzilov et al. to compare the performance of ncRNA finders [43].

ROC curve is defined as sensitivity vs false positive rate plot; sensitivity and false positive rate are defined as follows:

$$
\begin{aligned}
& \text { false positive rate }=(1-\text { specificity })=\frac{F P}{T N+F P}, \\
& \text { sensitivity }=\frac{T P}{T P+F N}, \quad \text { specificity }=\frac{T N}{T N+F P},
\end{aligned}
$$

where, $T P, F P, T N$ and $F N$ are the number of true positives, false positives, true negatives and false negatives, respectively. In the case of comparative ncRNA prediction, sensitivity indicates how many positive alignments (i.e. alignments containing true ncRNAs) are correctly predicted as ncRNA; false positive rate represents how many negative alignments are misclassified as ncRNA. For example, false positive rate $=1 \%$ means that one false positive is found when we evaluate 100 negative alignments.

\section{Genome sequences}

The genome sequences (excluding mitcondorial chromosome) of $S$. cerevisiae and the contigs of other six fungi ( $S$. bayanus, S. castellii, S. kluyveri, S. kudriavzevii, S. mikatae, and $S$. paradoxus) were downloaded at Saccharomyces Genome Database (SGD) [44]. Annotated fasta files for $S$. cerevisiae (orf_coding.fasta, rna _coding.fasta, NotFeature.fasta, and other_features_genomic.fasta) were also downloaded at SGD. We masked the genome sequences of S. cerevisiae according to the other_features_genomic.fasta file to remove repetitive sequences from the genome sequences.

\section{Pairwise comparison of genomic sequences}

To efficiently search for ncRNA candidates with low sequence identity, we focused on our scan to the relatively short ( $50 \mathrm{bp}$ to $2,000 \mathrm{bp}$ ) low-identity regions located between two regions which are conserved at sequence level. By exploring the regions neighboring such conserved regions, we can expect to find the ncRNAs hidden in a conserved synteny. The conserved regions were detected by using WU-BLAST [45] comparison (cutoff Evalue $=10^{-3}$ ) between $S$. cerevisiae and the other fugal genomes. Then we constructed 'target regions', which are the regions scanned by Cofolga2, as follows. First, the $S$. cerevisiae genome sequence was divided into intergenic (NotFeature), orf_coding, and rna_ coding sequences in accordance with the annotations in SGD [44]. Then target region was defined for each divided $S$. cerevisiae sequence as illustrated in Figure 4 if the divided sequence overlaps the low-identity region located between the conserved regions. As a result, the target regions which we obtained by the WU-BLAST comparison cover 2,196,982 bp of the S. cerevisiae genome (this corresponds to $18 \%$ of all auto chromosomes of S. cerevisiae).

The present approach for generating the target regions is similar to that used in the genome comparison between human and mouse by Torarinsson et al. [46]. Compared to their approach, however, ours is more conservative since it requires the target regions to be sandwiched by two conserved regions, while Torarinsson et al. scanned the regions neighboring to singly conserved regions. In other words, our definition is a subset of that of Torarinsson et al.. 


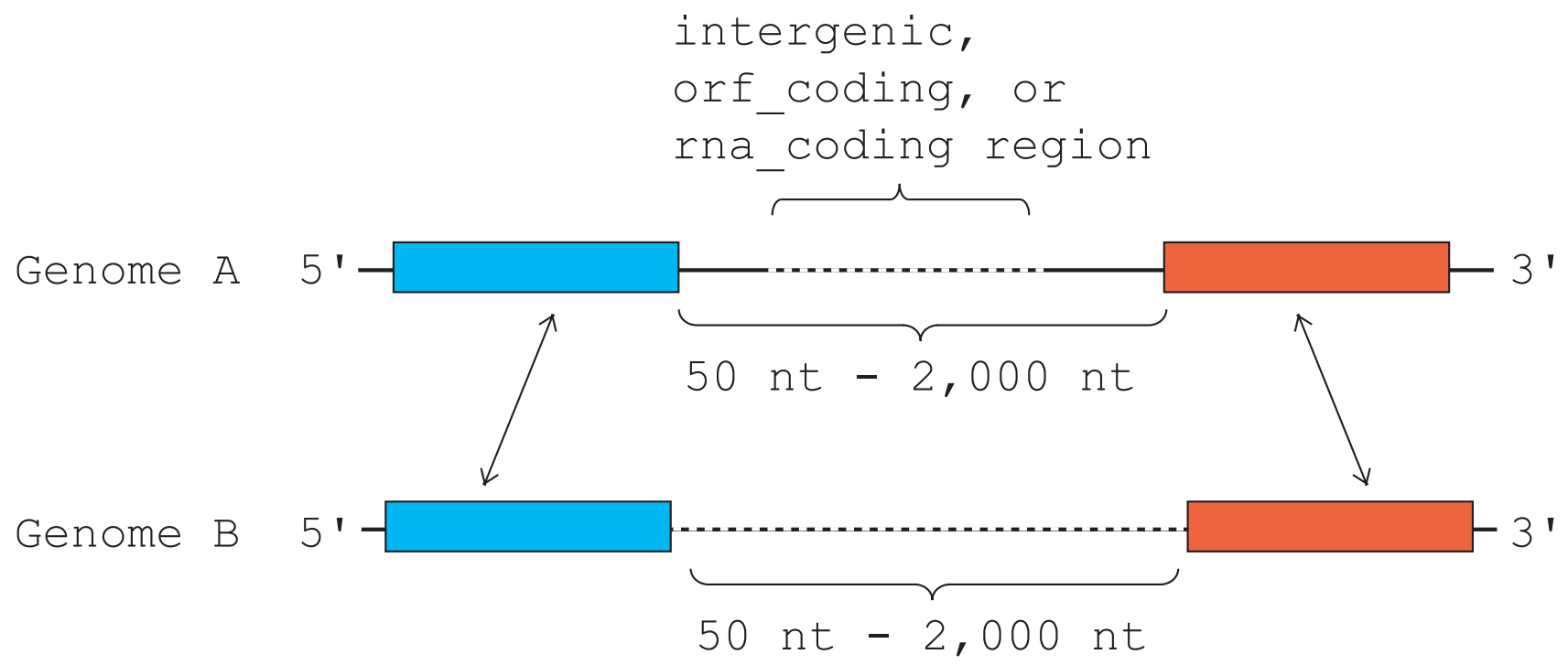

Figure 4

Definition of the target region to be scanned by Cofolga2. The conserved sequences are indicated by boxes, and matching sequences are represented by arrows. In this figure, Genome A conrresponds to S. cerevisiae, and Genome B corresponds to an other fungal genome. Dashed lines indicate the sequences belonging to the target region. A situation such that the arrows are crossing each other is not allowed. If a part of intergenic, orf_coding, or rna_coding region overlaps the conserved region, the overlapped part is excluded from the target region.

We scanned each target region using a dual sliding window according to the following procedure. Let us call the two genome sequences belonging to a target region genome A and genome B. Subsequences were generated by moving a sliding window of $150 \mathrm{nt}$ with a shift size of $50 \mathrm{nt}$ on each genome sequence, and then all-vs-all pairwise alignment between the subsequences of genome A and those of genome B was performed with Cofolga2. After the comparison, each pairwise alignment was processed by the trained SVM to assign a SVM classification probability to discriminate whether the pairwise alignment contains ncRNA candidates or not.

\section{Results and discussion}

Convergence test with respect to GA population size and iteration number

To know how the GA population size and iteration number affect the alignment quality, we studied the population size and iteration number dependence of the Cofolga2's performance, where we define population size $=$ iteration number to reduce the number of free parameters. Figure 5 shows the (mean SPS $) \times($ mean SCI) for the fourteen sequence pairs in Additional File 1 as a function of population size. As can be seen from the figure, the (mean SPS $) \times($ mean SCI) is almost saturated between population size 50 and 100. Based on this observation, we used population size ( $=$ iteration number $)=50$ for the benchmarks and ncRNA discovery in the present study.

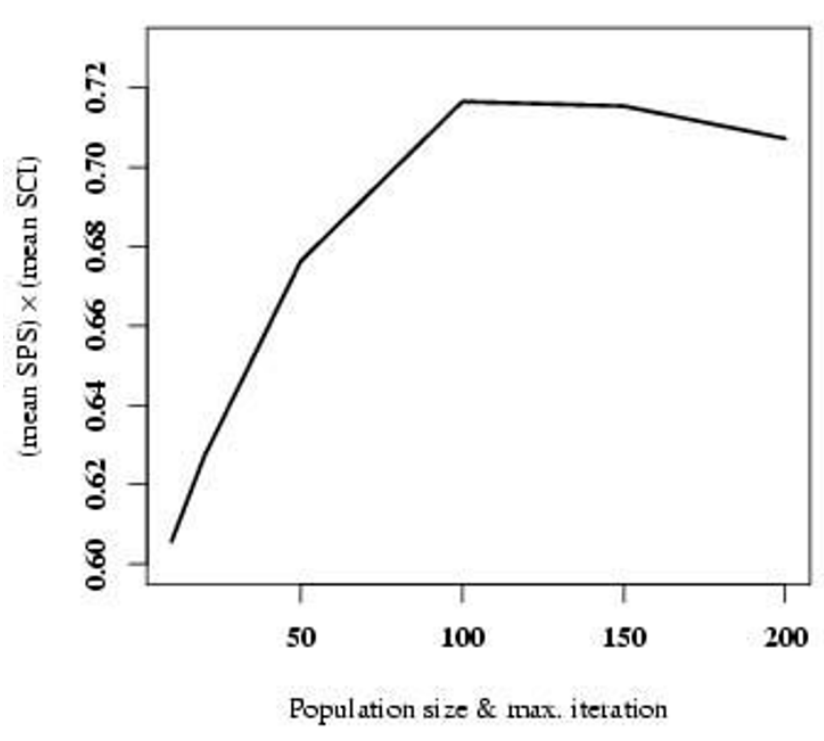

Figure 5

Convergence property with respect to GA population size and iteration number. This figure shows (mean SPS $) \times($ mean $\mathrm{SCl})$ as a function of GA population size (= iteration number). The means were taken over five runs with different initial random numbers. 


\section{RNA alignment benchmark and performance comparison} with other methods

Figure 6 shows the benchmark results for Cofogla2 and other programs for structural or non-structural sequence alignment. In this benchmark, 5,010 pairwise alignments ( $\leq 75 \%$ id) taken from the $\mathrm{k} 2$ dataset of BRAliBase 2.1 are used. The programs used for the comparison are summarized in Table 1. In this performance test, as can be seen from Figure 6, Cofolga2 outperformed the light-weighted programs (StrAl, LaRA, and LocARNA, and the non-structural alignment programs) at $\leq 50 \%$ id in both SPS and SCI. In addition, Cofolga 2 showed a performance comparable with the other structural RNA alignment programs in SCI and was the second-best method between 30\%id and 50\%id in SPS, where Foldalign revealed the best performance. When the fourteen training sequence pairs were excluded from the dataset, the identical conclusion was obtained.

Since GA is a sampling method utilizing random number, it is important to know how an initial random number affects the alignment quality. To examine random number dependence of Cofolga2, we performed five independent runs for the $\mathrm{k} 2$ dataset with different initial random numbers. As a result, we confirmed that the differences between the benchmark results due to the difference in initial random number are very small for a wide range of sequence identity (Additional File 2).

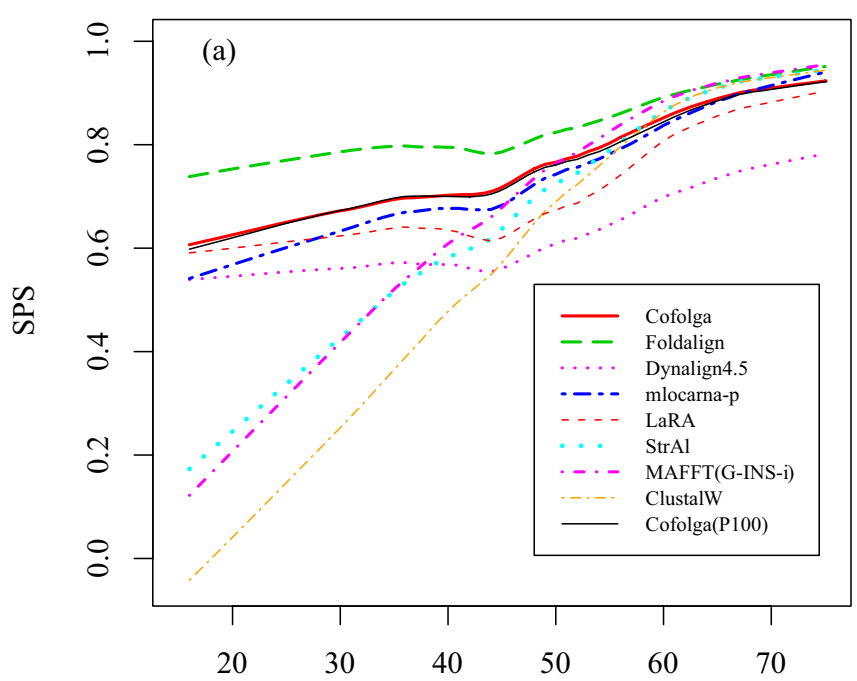

Sequence identity $(\%)$
In the benchmark with the ITS2 dataset, we found that Cofolga 2 showed the best performance (the averaged CCs for Cofolga2, Foldalign 2.1.0, and LocaRNA are 0.42, 0.30 , and 0.38 , respectively).

\section{Computational time and memory usage}

The computational times (including those for the BPP computation by RNAfold) measured for Cofolga2 and other structural RNA sequence alignment methods are shown in Figure 7 . The computational times were measured with a Xeon PC (2.4 GHz/3 GB RAM/Red Hat Linux 9.0).

For the RNA families shorter than approximately $85 \mathrm{nt}$, Foldalign showed computational times comparable with Cofolga2. For longer RNA families, Foldalign was much slower than Cofolga2 except for K_ chan_ RES (the data of $\mathrm{K}_{\text {_ }}$ chan_ RES can be found at $113 \mathrm{nt}$ in Figure 7). The computational times of Dynalign were in general much longer than the other methods in the present benchmark. In addition, the computational times of Foldalign and Dynalign were not scaled monotonically with respect to sequence length. This could be due to the pruning algorithm of Foldalign and the constraint used in Dynalign, i.e. when these accelerators do not work well the programs become slower.

LocARNA was faster than Cofolga2 up to approximately 150 nt. For longer RNA families, however, the computa-

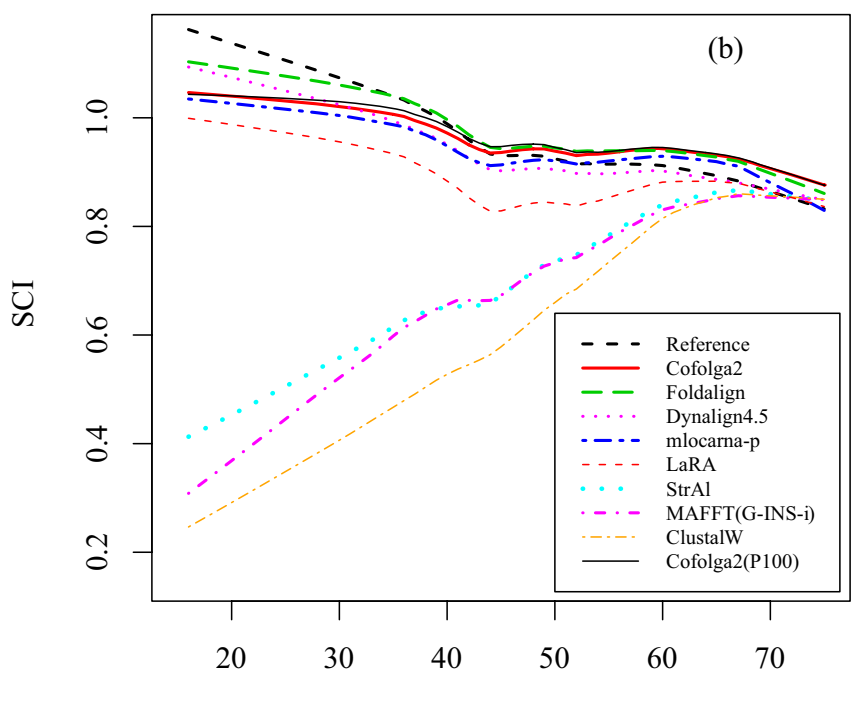

Sequence identity $(\%)$

\section{Figure 6}

RNA alignment benchmark with the $\mathbf{k 2}$ dataset of BRAliBase 2.I. Alignment quality benchmark results for (a) sum-ofpair score (SPS) and (b) structure conservation index (SCl). The results for Cofolga2 with a population size $=50$ and 100 are denoted by 'Cofolga' and 'Cofolga(PI00)', respectively. The curves were drawn by lowess smoothing with a smoother span of 0.3 . 


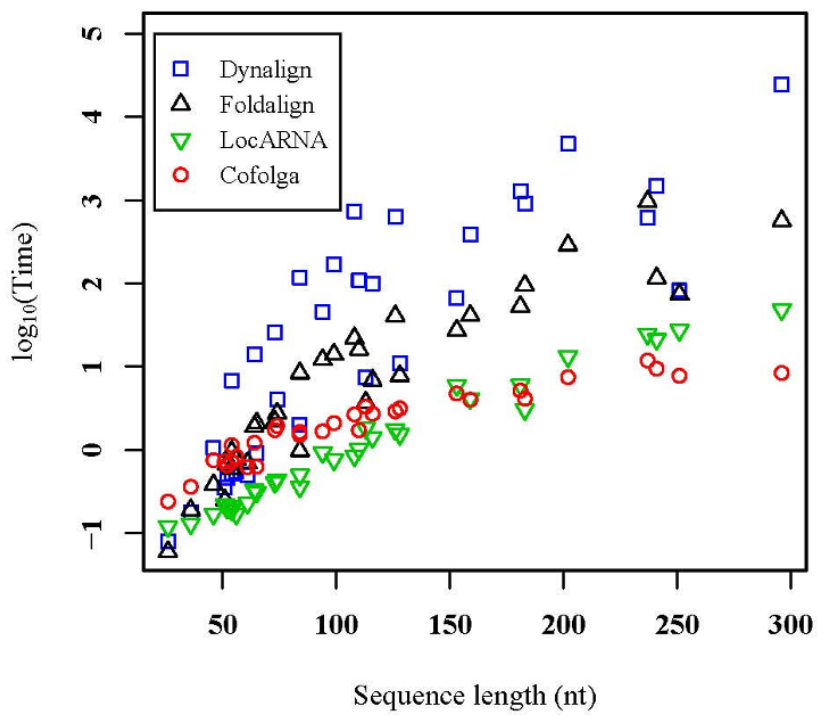

Figure 7

Comparison of computational times. Each symbol corresponds to the averaged computational time and averaged sequence length of one RNA family, where time is represented as $\log _{10}$ (time in seconds).

tional time of LocARNA became comparable with or longer than that of Cofolga2, e.g. average computational times of Cofolga2 and LocARNA for Cobalamin (202 nt) were $7.5 \mathrm{sec}$. and $13.2 \mathrm{sec}$., respectively.

To examine the memory usage of Cofolga2, we performed a structural alignment of two SRP _euk _arch RNAs, AP003253.3 (317 nt) and AC005275.1 (305 nt) taken from the BRAliBase $2.1 \mathrm{k} 2$-dataset (the file name of the sequence pair is included in Additional File 1). Since this computation is one of the largest calculations in the present study, we can estimate the upper bound of the memory usage from the result. Consequently, we found that Cofolga2 needs only 10.0 MB RAM to perform the calculation. This memory usage is smaller than or comparable with those of other latest structural RNA sequence alignment methods. According to literature [47], Foldalign 2.1.0, Dynalign 4.5, and LocARNA 0.99 need at least 17.3, 13.3, and 7.6 MB RAM, respectively, to align the 5S rRNAs with an average sequence length of 119.4 nucleotides.

\section{SVM training results and ncRNA prediction benchmark}

In Figure 8, ROC curves by Cofolga2, RNAz, and Dynalign are plotted. To make the plot, first we ran each alignment program for the SVM test data, and then extracted the pairwise alignments satisfying (alignment length after removing gapped columns) $\geq 50 \mathrm{nt}$ and $\% \mathrm{id} \leq 50 \%$, where the sequence identity based on the BRAliBase 2.1 alignments was used. As a consequence, each ROC curve was drawn

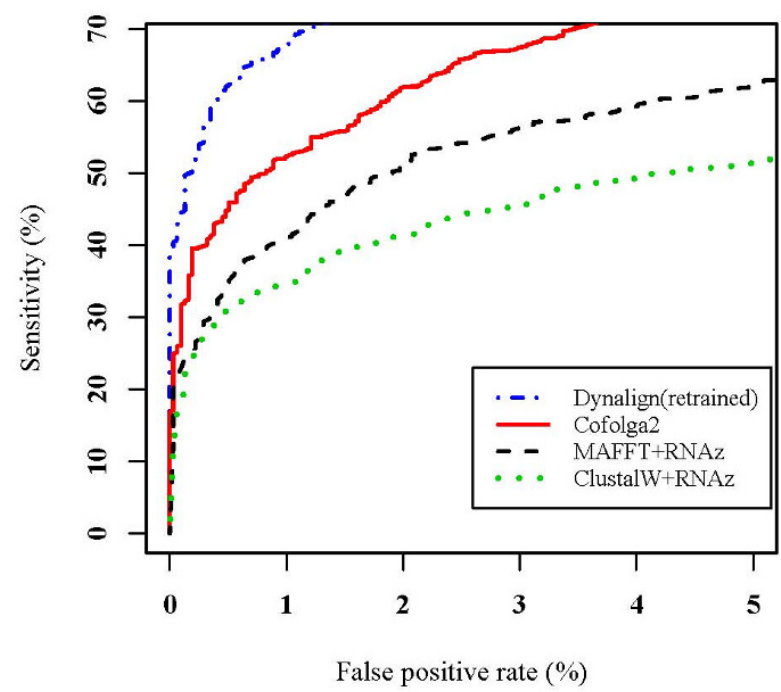

Figure 8

Comparison of ncRNA prediction performance. The ROC curves drawn for the alignments $\leq 50 \%$ id. The ROC curve obtained using Dynalign 4.3 with the SVM model retrained in the present study is denoted by 'Dynalign(retrained)'.

based on approximately 4,700 RNA alignments. In Figure 8, "ClustalW+RNAz" indicates that an alignment is constructed by ClustalW and then the alignment is evaluated by $\mathrm{RNAz}$ to predict whether the alignment contains ncRNA candidates or not. We used ClustalW and MAFFT to construct input pairwise alignments for RNAz, since ClustalW is the standard sequence alignment program and MAFFT is the best non-structural sequence alignment method in accordance with the previous benchmark performed with BRAliBase 2.1 [37]. In our comparison, we ran Dynalign 4.3 (maximum separation parameter $M=8$ was used) not with the original SVM model trained in [43], but with a SVM model which was re-trained with the training dataset for the SVM model of Cofolga2. This is because the original SVM model of Dynalign was trained with only tRNA and $5 S$ rRNA sequences, and the Dynalign with the original SVM model showed a poor prediction performance (data not shown) in our benchmark where more RNA families are included. In addition, we did not include Foldalign in the ncRNA prediction benchmark using BRAliBase 2.1, since the ncRNA prediction by Foldalign needs flanking sequences of a ncRNA sequence to obtain a statistical value [48], and the ncRNA sequences of BRAliBase 2.1 do not have flanking sequences. As can be seen from Figure 8, Cofolga2 outperformed RNAz when sequence identity is lower than $50 \%$. Although the retrained Dynalign showed better prediction results compared to Cofolga2, Dynalign was much slower compared to Cofolga2. 
When one performs a genomic scan, it is important to use a cutoff value which gives a very low false positive rate, since genome-scale calculations usually process a number of sliding windows containing negative data. To reduce the false positive rate as small as possible, we chose a cutoff $P_{S V M}=0.9$ whose sensitivity and false positive rate are $25.3 \%$ and $0.06 \%$, respectively. Cofogla2 with this cutoff $P_{S V M}$ gives a better false positive rate compared to RNAz (e.g. the false positive rate of MAFFT+RNAz was 2.9 times larger than that of Cofolga2 at sensitivity $=27.5 \%$ ).

\section{Comparative prediction of yeast ncRNAs}

We obtained 6,349 target regions whose average sequence lengths for $S$. cerevisiae and the other fungi are $446 \mathrm{bp}$ and 980 bp, respectively. These target regions cover 2,196,982 bp of S.cerevisiae and $2,885,670$ bp of the other fungi. After processing the 2,383,802 sequence pairs (generated from the target regions using a dual sliding window) by Cofolga2 and the SVM we trained, we obtained 2,807 pairwise alignments which have SVM probabilities $\geq 0.9$. The $S$. cerevisiae sequences of the obtained pairwise alignments were clustered into 'ncRNA candidate regions' by a single linkage clustering, where overlapped or neighboring sequences are clustered. The ncRNA search in yeast (with a PC cluster consisting of thirteen Pentium4 PCs) took approximately twenty days. The obtained candidate regions are summarized in Table 2 . As shown in the table, we found ncRNA candidates at 714 intergenic regions, 1,311 protein-coding regions, and twenty known ncRNA regions in the $S$. cerevisiae genome. Based on the total number of alignments processed by Cofolga 2 and the false positive rate $(0.06 \%)$ obtained in the benchmark, we estimated the number of false positive alignments $=1430$, leading to (the estimated number of false positive alignments $) /($ the number of alignments predicted as ncRNA) $=$ $51 \%$. This value is almost same with the corresponding value obtained in the human ncRNA finding by CMfinder [24].

In the present predictions, we obtained 53 intergenic regions, 43 protein-coding regions, and 12 known ncRNA regions as ncRNA candidates (Table 3), which overlap at least one of the previous RNAz and QRNA predictions; where we classified a candidate as an "overlapped" region if $\geq 10 \%$ of the nucleotides of the candidate overlaps an RNAz or QRNA prediction. Relatively small overlaps between our ncRNA candidates and those by RNAz and QRNA are not surprising because our method does not require sequence conservation of ncRNA candidates while RNAz and QRNA directly utilize the sequence similarity between ncRNA candidates. For example, the lowest sequence identity in the alignments containing our ncRNA candidates was 15\% (Figure 9).
Table 2: Summary of the predicted S. cerevisiae ncRNA candidates

\begin{tabular}{llccc}
\hline$P_{\text {cutoff }}$ & organism & int & $\begin{array}{c}\text { S. cerevisiae } \\
\text { orf }\end{array}$ & rna \\
\hline \multirow{2}{*}{0.90} & S. mikatae & $252(27,760)$ & $356(40,529)$ & $10(786)$ \\
& S. kudriavzevii & $247(28262)$ & $423(49,395)$ & $9(692)$ \\
S. bayanus & $116(12,230)$ & $221(26,143)$ & $3(279)$ \\
S. castellii & $105(12,537)$ & $260(30,009)$ & $0(0)$ \\
S. kluyveri & $184(18,904)$ & $315(36,471)$ & $9(460)$ \\
S. paradoxus & $118(12,493)$ & $177(22,219)$ & $2(200)$ \\
all & $714(102,652)$ & $1,311(197,116)$ & $20(2,267)$ \\
\hline \multirow{2}{*}{0.95} & & & \\
& S. mikatae & $83(10,234)$ & $108(14,190)$ & $3(350)$ \\
S. kudriavzevii & $76(9,862)$ & $121(15,905)$ & $2(256)$ \\
S. bayanus & $37(4,692)$ & $63(8,109)$ & $0(0)$ \\
S. castellii & $32(3,886)$ & $79(10,532)$ & $0(0)$ \\
S. kluyveri & $55(6,566)$ & $88(11,564)$ & $2(210)$ \\
S. paradoxus & $30(3,497)$ & $47(6,286)$ & $1(150)$ \\
all & $253(37,128)$ & $435(64,974)$ & $7(966)$ \\
\hline
\end{tabular}

The 'int', 'orf', and 'rna' columns indicate the number of ncRNA candidate regions for the $S$. cerevisiae intergenic, orf-coding, and known RNA sequences, respectively. In parenthesis, their total length $(\mathrm{nt})$ is shown. The 'organism' column indicates the counterpart of each genome comparison. The 'all' rows are the summary for all genome comparisons after eliminating positional overlaps. $P_{\text {cutoff }}$ is a cutoff value for the SVM classification probability. In this table, the results for $P_{\text {cutoff }}=0.9$ and $P_{\text {cutoff }}=0.95$ are shown.

Figure 10 shows the histogram of the GC content for all ncRNA candidates we predicted. In the previous comparative ncRNA predictions [5] in which RNAz and EvoFold

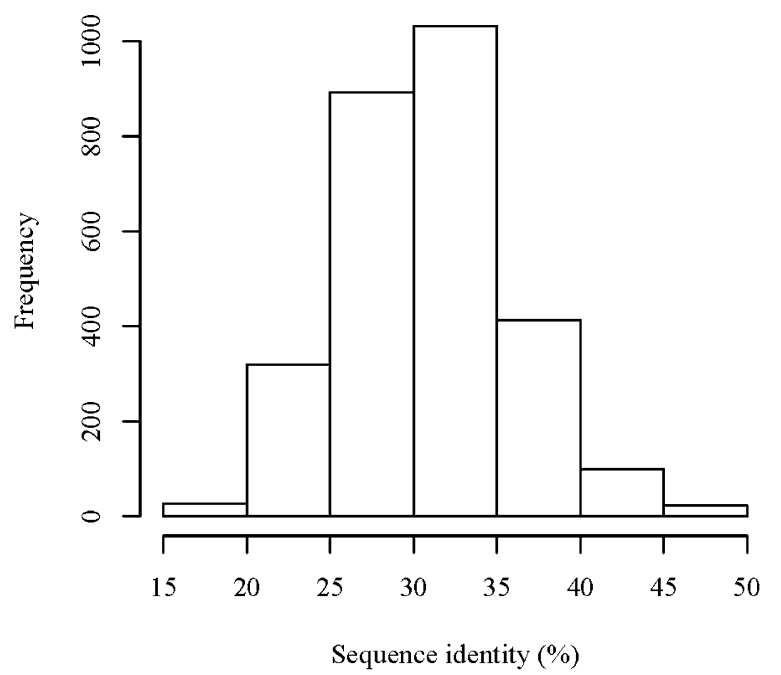

Figure 9

Sequence identity distribution for the predicted ncRNA candidates. The sequence identity distribution of the alignments with a significant SVM classification probability $(\geq 0.9)$. 
Table 3: Overlaps between the predicted ncRNA candidates and the previsous predictions

\begin{tabular}{cccc}
\hline region & RNAz & QRNA & Cofolga \\
\hline intergenic & $39(5,941)$ & $24(3,556)$ & $657(94,705)$ \\
coding & $45(6,904)$ & $0(0)$ & $1,266(191,052)$ \\
known RNAs & $11(1,378)$ & $12(1,328)$ & $6(639)$ \\
\hline
\end{tabular}

The 'RNAz' and 'QRNA' columns indicate the number of the present S. cerevisiae ncRNA candidates overlapping with those by RNAz [49] and QRNA [4], respectively. In parenthesis, the total length (nt) for each region is mentioned. When $\geq 10 \%$ of the nucleotides of a ncRNA candidate overlaps the RNAz or QRNA prediction, we included the ncRNA candidate in the overlapped ncRNA candidates. The 'Cofolga' column indicates the ncRNA candidates predicted by Cofolga2 alone, i.e. novel ncRNA candidates obtained in the present study. Since the ncRNA candidate list of QRNA (Table SI of [4]) does not contain strand information, we took only positional overlap into account when we examine the overlaps between the present ncRNA candidates and those by QRNA.

have been used to predict human ncRNAs, it was found that there are biases in the base composition distribution of the predicted ncRNA sequences, i.e. RNAz favors GCrich sequences, while Evofold tends to predict AU-rich ones as ncRNA candidates [49]. As indicated in Figure 10, our prediction result is not biased in GC content (the average GC content of whole DNA sequences of $S$. cerevisiae auto chromosomes is approximately $37 \%$ ).

The target regions we obtained includes 82 known ncRNAs. Of these, eighteen loci were included in the alignments with $P_{S V M} \geq 0.9$. The detail of the predicted

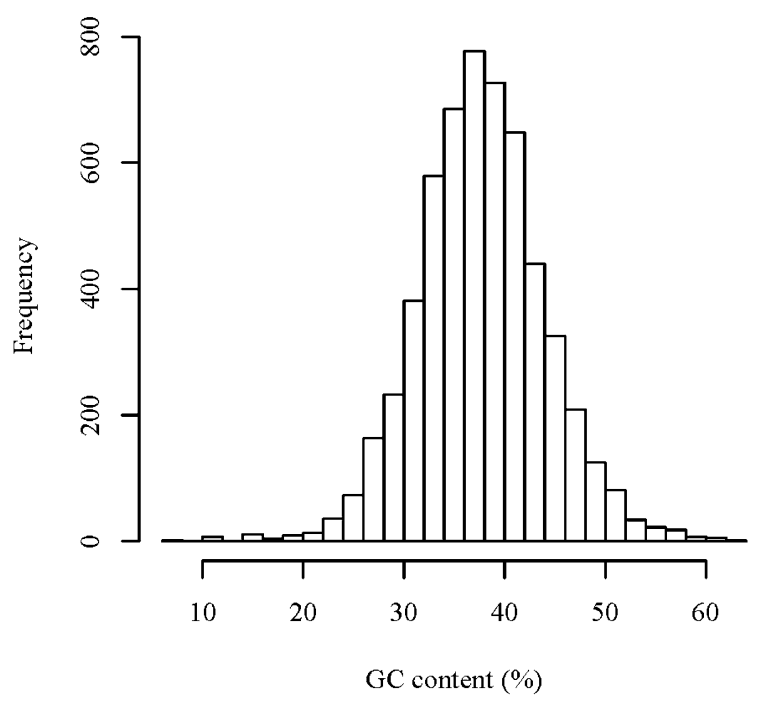

Figure 10

Histogram of the GC content for the yeast ncRNA candidates. The GC content of the $S$. cerevisiae ncRNA candidates distributed around the average GC content (approximately $37 \%$ ) of $S$. cerevisiae auto chromosomes. known ncRNAs is summarized in Additional File 3. An estimated sensitivity for the ncRNA prediction calculated based on this observation is approximately $22 \%$. It is noteworthy that Cofolga 2 correctly predicted the strand of fifteen known ncRNAs, i.e. correct strand was assigned to approximately $83 \%$ of the eighteen known ncRNAs. In these strand predictions, we adopted the strand with the highest $P_{S V M}$ when $P_{S V M} \geq 0.9$ was assigned to the both strands.

Table 4 shows how many ncRNA candidates overlap the loci of the experimentally determined transcripts. In the table, experimental data taken from tilling array [50-52] and cDNA [53] are included. Here we found that the genomic positions of the results by David et al. and those by Miura et al. significantly ( $\geq 50 \%$ of the nucleotides of a candidate) overlap 112 and 69 ncRNA candidates of the present study, respectively, while our results show fewer overlaps with the transcripts by Davis et al. and Samanta et al.. Consequently, it turned out that 176 intergenic ncRNA candidates (this corresponding to approximately $25 \%$ of our intergenic candidates of S. cerevisiae) have at least one experimental support for their expression. By comparing the genomic positions of our results and the annotation (orf_coding.fasta) of SGD, we found that 95 and 71 intergenic candidates are located within $120 \mathrm{bp}$ from the 5'-end and 3'-end of a CDS, respectively. In addition, eight intergenic candidates (SC000040I, SC000063I, SC000083I, SC000157I, SC000233I, SC000331I, SC000485I, SC000531I) are found at within $120 \mathrm{bp}$ from the 5 '- and 3'-ends of two CDSs, i.e. these eight candidates are sandwiched by two protein-coding genes. It is noted that the $5^{\prime}$ and $3^{\prime}$ ends of our ncRNA candidates can have an ambiguity of a few tens of nucleotides due to the gapped 5' and/or 3' edges of the pairwise alignments.

Recently, the ncRNAs found in protein-coding regions have been reported. In our prediction, we obtained more than one thousand ncRNA candidates in protein-coding regions. In these, 628 candidates are predicted at sense strand, and 684 candidates were predicted at antisense strand. One ncRNA candidate (SC000407F) simultaneously overlaps two protein-coding genes as sense and antisense since these two protein-coding genes overlap each other.

The detail of our prediction results and annotations can be browsed at our web server [40] in which the prediction results are retrieved through MySQL queries.

\section{ncRNA candidates conserved among multiple sequences}

By manually inspecting our prediction results, we found four intriguing examples containing conserved secondary structures across multiple species/sequences which have characteristic secondary structures in spite of their low 
Table 4: Overlaps between the predicted ncRNA candidates and experimentally determined transcripts

\begin{tabular}{cccccc}
\hline region & David et al. & Davis et al. & Miura et al. & Samanta et al. & all \\
\hline intergenic & $112(16,516)$ & $37(5,370)$ & $69(9,938)$ & $2(239)$ & $176(25,682)$ \\
coding & $693(109,508)$ & $31(4,454)$ & $210(32,721)$ & $0(0)$ & $738(116,286)$ \\
known RNA & $7(939)$ & $8(958)$ & $5(887)$ & $0(0)$ & $12(1,577)$ \\
\hline
\end{tabular}

The number of the predicted ncRNA candidates which overlap the loci of experimentally determined transcripts in literature. In parenthesis, the total length (nt) for each region is mentioned. Since the transcript list provided by Davis et al. (Supporting Table I in [5I]) does not contain strand information, we took only positional overlap into account when we examine the overlaps between the present ncRNA candidates and the transcripts reported by Davis et al..

average sequence identities. Figure 11 shows the alignment and structure of an intergenic $S$. cerevisiae sequence and two paralogous sequences of $S$. mikatae taken from the ncRNA candidate SC000056I. Since genomic separation between these two $S$. mikatae sequences are small (302 bp), these two $S$. mikatae sequences are a possible ncRNA cluster. Figure 12 shows the $S$. cerevisiae sequence of an intergenic ncRNA candidate (SC000383I) which was found at 39,052 bp to 39,158 bp of chromosome 3 and aligned with the sequences of $S$. mikatae and $S$. paradoxus. As can be seen from figures 11 and 12, these ncRNA candidates reveal characteristic consensus secondary structures in spite of their low average pair sequence identities (31\%id for SC000056I and 28\%id for SC000383I).

The ncRNA candidate SC000983F is one of the longest regions predicted in the present study. This candidate contains a consensus secondary structure motif shared by three species (Figure 13). In addition to the structure shared by three species, ncRNA candidate SC000983F contains a relatively long (300 bp) secondary structure (Additional File 4) conserved between two species (S. cerevisiae and $S$. kudriavzevii). It is noteworthy that almost all sequences contained in SC000983F are antisense sequences. The sequences of $S$. cerevisiae are antisense sequences of a gene CLN1 coding G1 cyclin which is involved in regulation of the cell cycle, and the sequences of S. kluyveri and S. kudriavzevii are antisense sequences of predicted ORFs according to the SGD annotation. These results imply that this ncRNA candidate is a functional antisense ncRNA with characteristic secondary structures.

The $S$. cerevisiae sequence of ncRNA candidate SC01074F is located at the sense strand of PMS1, a verified ORF coding an ATP-binding protein. The sequences of $S$. paradoxus and $S$. bayanus, which overlap the predicted ORFs of each genome according to the SGD annotation, are structurally aligned to the S. cerevisiae sense sequence in SC01074F (Figure 14). These sequences are new candidates of functional RNA secondary structure within a coding region such as the localization elements of ASH1 which do not show sequence conservation but harbor conserved RNA secondary structure [54]. The multiple alignments for SC000056I, SC000383I, SC000983F, and SC001074F were constructed by manual operation (including partial re-alignment by a progressive alignment with Cofolga2) based on the pairwise alignments by Cofolga2. Since the progressive multiple alignment using Cofolga2 has not fully tested yet, we didn't benchmark it in this paper. The figures of consensus secondary structure and alignment were drawn by processing the multiple alignments at RNAalifold web server [34].

The examples described in this section (SC000056I, SC000383I, SC000983F, and SC01074F) have at least one experimental evidence for their expression according to the tilling array/cDNA data in literature [50-53].

\section{Conclusion}

As can be known from a number of recent papers describing various structural RNA sequence alignment programs, it is a difficult problem to find a good RNA alignment with low sequence conservation. In the present study, we developed a new efficient GA for constructing structural RNA pairwise alignment with a new objective function and random alignment generation algorithm. The new GA is accurate and efficient in both time and memory usage, hence we applied it to the comparative ncRNA discovery between $S$. cerevisiae and related species using a SVM trained with the sequences and alignments taken from BRAliBase 2.1. As a result, we successfully obtained ncRNA candidates located at 714 intergenic regions and 1,311 protein-coding regions including antisense sequences, $>92 \%$ of which is novel candidates since they show no overlaps with the genomic positions of the previous predictions and known ncRNAs. Indeed, our approach is not suitable for identifying all ncRNA sequences embedded in a genome, it gives a valuable tool complementary to the sequence-alignment-based ncRNA finders such as RNAz and QRNA, since the present method often found the ncRNA candidates which cannot be found by such sequence-alignment-based ncRNA finders. The results of the present study indicate that still a number of structured RNA transcripts with significant structural and evolutional signals is hidden in genomic sequences, and further exploration for novel ncRNAs using computational methods is inevitable to unveil the RNomics of genomes. 


\section{SC000056I}
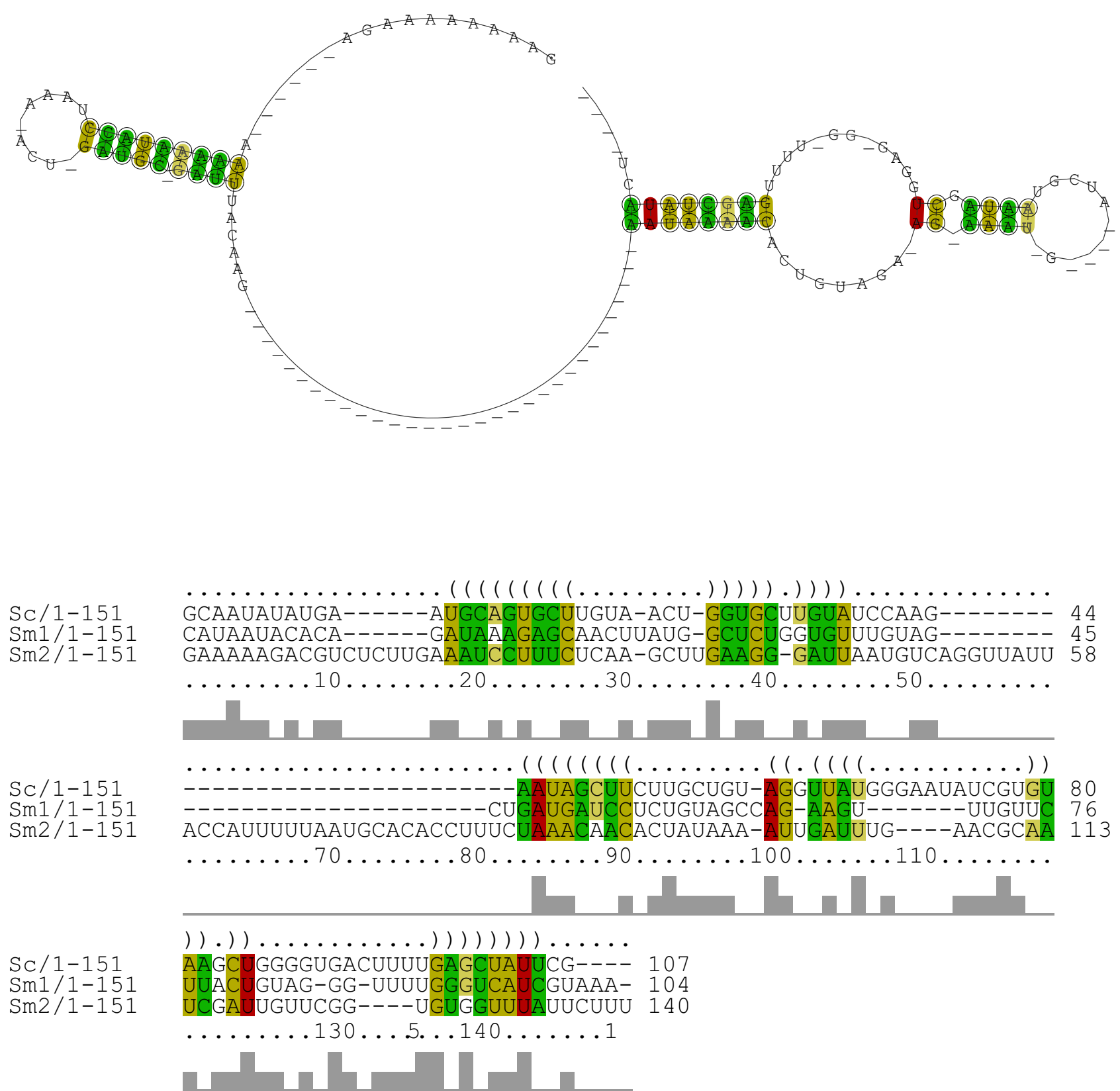

\section{Figure II}

Consensus structure and alignment of ncRNA candidate SC000056I. This alignment is composed of a $S$. cerevisiae sequence (chr3/39,052-39,I58 bp/+) and two S. mikatae sequences (c260l/5,7/2-5,86I bp/+ and c260I/6I62-63 II bp/+, denoted by $\mathrm{SmI}$ and $\mathrm{Sm} 2$, respectively). The averege pair sequence identity of this alignment is $31 \%$. 


\section{SC000383I}

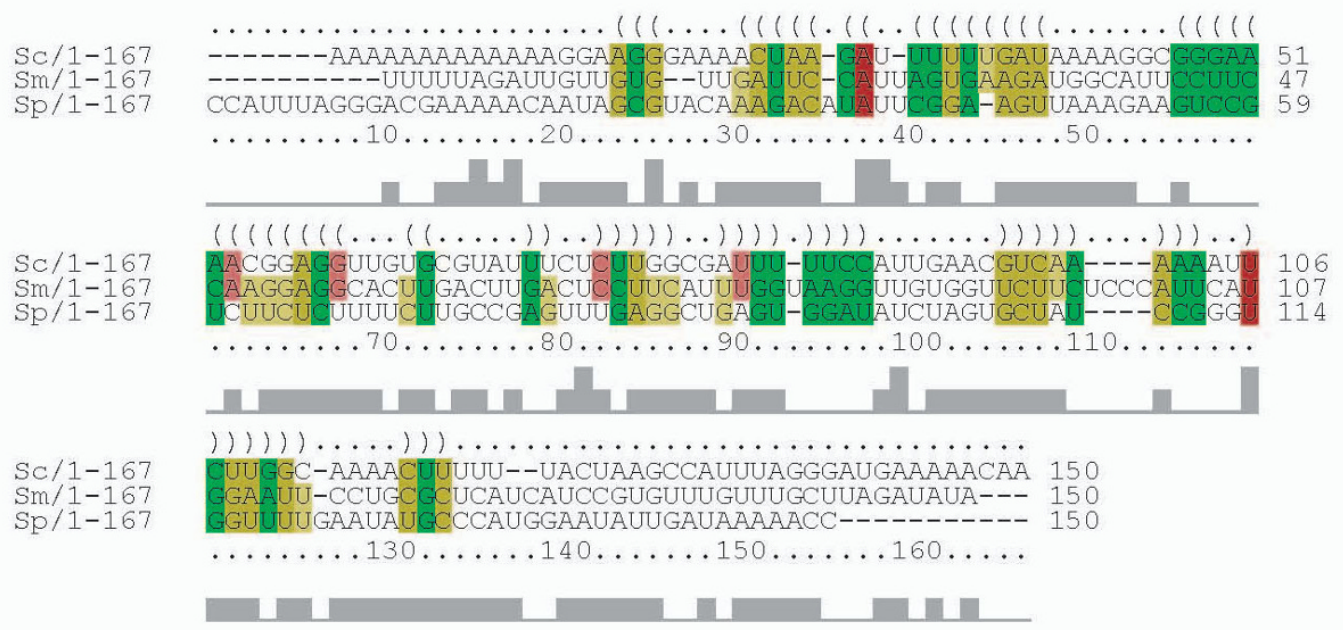

\section{Figure 12}

Consensus structure and alignment of ncRNA candidate SC 0003831 . This alignment is composed of a $S$. cerevisiae sequence (chr I0/550,008-550, I57 bp/-), a S. mikatae sequence (c27/2/2,245-2,394 bp/-), and a S. paradoxus sequence (c303/ $5,880-6,029 \mathrm{bp} /-)$. The averege pair sequence identity of this alignment is $28 \%$. 

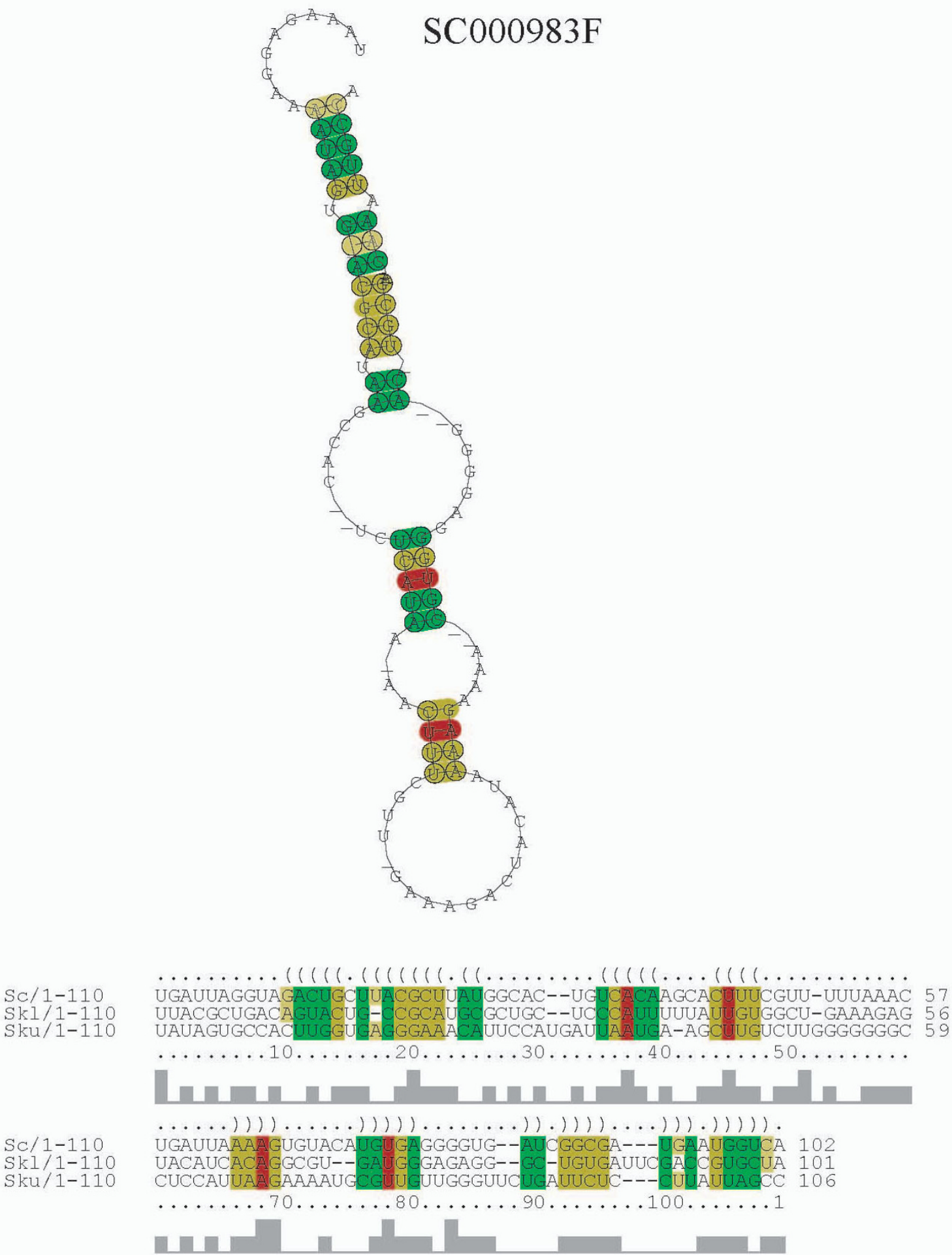

\section{Figure 13}

Consensus structure and alignment found in ncRNA candidate SC000983F. This alignment is composed of a S. cerevisiae sequence (chrl3/663,965-664,066 bp/-), a S. kluyveri sequence (c2074/4,854-4,954 bp/-), and a S. kudriavzevii sequence (c2037/10,962-II,067 bp/-). The averege pair sequence identity of this alignment is $27 \%$. 


\section{SC001074F}
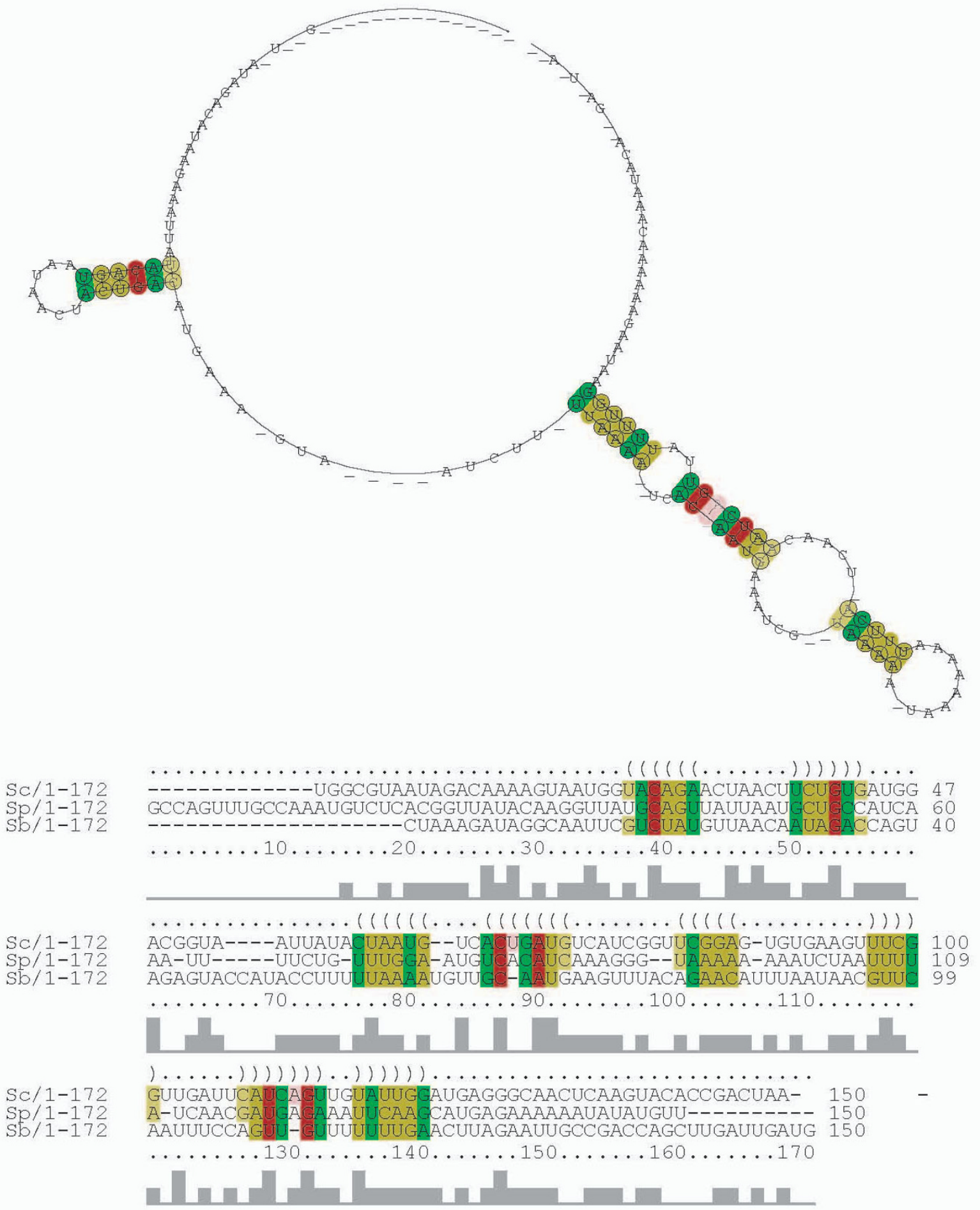

$\mathrm{Sc} / 1-172$

\section{Figure 14}

Consensus structure and alignment of ncRNA candidate SC00I 074F. This alignment is composed of a S. cerevisiae sequence (chrl4/474,642-474,79| bp/+), a S. paradoxus sequence (c250/6,937-7,086 bp/+), and a S. bayanus sequence (c627/ $32,967-33,116 \mathrm{bp} /+)$. The averege pair sequence identity of this alignment is $34 \%$. 


\section{Availability and requirements}

Non-profit, academic users can download and use the executable files at the Cofolga2 website [40].

\section{Additional material}

\section{Additional File 1}

The file names of the RNA sequence pairs used in the GA parameter determination and memory usage test. The top fourteen files were used for the GA parameter determination and convergence test. The other one (denoted by ${ }^{*}$ ) was used only for examining the maximum memory usage. Click here for file

[http://www.biomedcentral.com/content/supplementary/14712105-9-521-S1.pdf]

\section{Additional File 2}

Initial random number dependence of the benchmark result. The benchmark results for the BRAliBase2.1 k2-dataset with five different initial random numbers. The results denoted by $\mathrm{R}=12345$ are same with those of Figure 2, since it is a default value. In addition, this figure includes the result obtained with a larger population size $(=100)$ with $\mathrm{R}$ $=12345$.

Click here for file

[http://www.biomedcentral.com/content/supplementary/14712105-9-521-S2.pdf]

\section{Additional File 3}

Known ncRNAs predicted in the present comparative genomics. The 'ID' column indicates the index assigned for the predicted ncRNA candidates in the present study. The 'Same/Diff' column shows whether the strand of known RNAs are correctly predicted or not, where 'Same' and 'Diff' indicate "strand is correctly predicted" and "not correctly predicted", respectively. For snR63 and NME1, both strands are predicted as ncRNA candidates. The 'Chr.', 'begin', 'end', and 'strand' columns give the genomic positions and strand of the ncRNA candidates. The 'RNA name', 'SGDID', and 'SGD annotation' columns correspond to ncRNA gene names, IDs, and annotations given in SGD, respectively.

Click here for file

[http://www.biomedcentral.com/content/supplementary/14712105-9-521-S3.pdf]

\section{Additional File 4}

Consensus structure and alignment of a sequence pair taken from ncRNA candidate SC000983F. This alignment is composed of a sequence of S. cerevisiae (chr13/663,564-663,863 bp/-) and a sequence of S. kudriavzevii (c2037/10,520-10,819 bp/-). The sequence identity of this alignment is $32 \%$.

Click here for file

[http://www.biomedcentral.com/content/supplementary/14712105-9-521-S4.pdf]

\section{Acknowledgements}

This work was partially supported by the Ministry of Education, Science, Sports and Culture, Grant-in-Aid for Young Scientists (B), 17700289, 20052006, Grant-in-Aid for Young Scientists (B), 19700272, 2007-2008, Grantin-Aid for Scientific Research (B), 18380028, 2006-2008, and a "Grant for Priority Research Designated by the President of Hirosaki University".

\section{References}

I. Carninci P, Kasukawa T, Katayama S, Gough J, Frith M, Maeda N Oyama R, Ravasi T, Lenhard B, Wells C, Kodzius R, Shimokawa K, Bajic V, Brenner S, Batalov S, Forrest A, Zavolan M, Davis M, Wilming L, Aidinis V, Allen J, Ambesi-Impiombato A, Apweiler R, Aturaliya R, Bailey T, Bansal M, Baxter L, Beisel K, Bersano T, Bono H, Chalk A, Chiu K, Choudhary V, Christoffels A, Clutterbuck D, Crowe M, Dalla E, Dalrymple B, de Bono B, Della Gatta G, di Bernardo D, Down T, Engstrom P, Fagiolini M, Faulkner G, Fletcher C, Fukushima T, Furuno M, Futaki S, Gariboldi M, Georgii-Hemming P, Gingeras T, Gojobori T, Green R, Gustincich S, Harbers M, Hayashi Y, Hensch T, Hirokawa N, Hill D, Huminiecki L, lacono M, Ikeo K, Iwama A, Ishikawa T, Jakt M, Kanapin A, Katoh M, Kawasawa Y, Kelso J, Kitamura H, Kitano H, Kollias G, Krishnan S, Kruger A, Kummerfeld S, Kurochkin I, Lareau L, Lazarevic D, Lipovich L, Liu J, Liuni S, McWilliam S, Madan Babu M, Madera M, Marchionni L, Matsuda H, Matsuzawa S, Miki H, Mignone F, Miyake S, Morris K, Mottagui-Tabar S, Mulder N, Nakano N, Nakauchi $\mathrm{H}, \mathrm{Ng}$ P, Nilsson R, Nishiguchi S, Nishikawa S, Nori F, Ohara O, Okazaki Y, Orlando V, Pang K, Pavan W, Pavesi G, Pesole G, Petrovsky N, Piazza S, Reed J, Reid J, Ring B, Ringwald M, Rost B, Ruan Y, Salzberg S, Sandelin A, Schneider C, Schonbach C, Sekiguchi K, Semple C, Seno S, Sessa L, Sheng Y, Shibata Y, Shimada H, Shimada K, Silva D, Sinclair B, Sperling S, Stupka E, Sugiura K, Sultana R, Takenaka Y, Taki K, Tammoja K, Tan S, Tang S, Taylor M, Tegner J, Teichmann S, Ueda $H$, van Nimwegen E, Verardo R, Wei C, Yagi K, Yamanishi H, Zabarovsky E, Zhu S, Zimmer A, Hide W, Bult C, Grimmond S, Teasdale R, Liu E, Brusic V, Quackenbush J, Wahlestedt C, Mattick J, Hume D, Kai C, Sasaki D, Tomaru Y, Fukuda S, KanamoriKatayama M, Suzuki M, Aoki J, Arakawa T, lida J, Imamura K, Itoh M, Kato T, Kawaji H, Kawagashira N, Kawashima T, Kojima M, Kondo S, Konno H, Nakano K, Ninomiya N, Nishio T, Okada M, Plessy C, Shibata K, Shiraki T, Suzuki S, Tagami M, Waki K, Watahiki A, OkamuraOho Y, Suzuki H, Kawai J, Hayashizaki Y: The transcriptional landscape of the mammalian genome. Science 2005, 309:I559- 1563.

2. Lu C, Tej S, Luo S, Haudenschild C, Meyers B, Green P: Elucidation of the small RNA component of the transcriptome. Science 2005, 309: 1567-1569.

3. Washietl S, Hofacker I, Stadler P: Fast and reliable prediction of noncoding RNAs. Proc Natl Acad Sci USA 2005, 102:2454-2459.

4. Rivas E, Eddy S: Noncoding RNA gene detection using comparative sequence analysis. BMC Bioinformatics 200I, 2:8.

5. Pedersen J, Bejerano G, Siepel A, Rosenbloom K, Lindblad-Toh K, Lander E, Kent J, Miller W, Haussler D: Identification and classification of conserved RNA secondary structures in the human genome. PLoS Comput Biol 2006, 2:e33..

6. Rivas E, Klein R, Jones T, Eddy S: Computational identification of noncoding RNAs in E. coli by comparative genomics. Curr Biol 200I, II:1369-1373.

7. Washietl S, Hofacker I, Lukasser M, Huttenhofer A, Stadler P: Mapping of conserved RNA secondary structures predicts thousands of functional noncoding RNAs in the human genome. Nat Biotechnol 2005, 23: 1383-1390.

8. Missal K, Zhu X, Rose D, Deng W, Skogerbo G, Chen R, Stadler P: Prediction of structured non-coding RNAs in the genomes of the nematodes Caenorhabditis elegans and Caenorhabditis briggsae. J Exp Zool B Mol Dev Evol 2006, 306:379-392.

9. Missal K, Rose D, Stadler P: Non-coding RNAs in Ciona intestinalis. Bioinformatics 2005, 2 I(Suppl 2):i77-78.

10. Rose D, Hackermuller J, Washietl S, Reiche K, Hertel J, Findeiss S, Stadler P, Prohaska S: Computational RNomics of drosophilids. BMC Genomics 2007, 8:406.

II. Sankoff D: Simultaneous solution of the RNA folding, alignment and protosequence problems. SIAM J Appl Math 1985, 45:810-825.

12. Havgaard J, Torarinsson E, Gorodkin J: Fast pairwise structural RNA alignments by pruning of the dynamical programming matrix. PLoS Comput Biol 2007, 3:1896-1908.

13. Harmanci A, Sharma G, Mathews D: Efficient pairwise RNA structure prediction using probabilistic alignment constraints in Dynalign. BMC Bioinformatics 2007, 8:130.

14. Kiryu H, Tabei $Y$, Kin T, Asai K: Murlet: a practical multiple alignment tool for structural RNA sequences. Bioinformatics 2007, 23:1588-1598.

15. Will S, adn IL, Hofacker KR, Stadler PF, Backofen R: Inferring Noncoding RNA Families and Classes by Means of Genome-Scale Structure-Based Clustering. PLoS Comp Biol 2007, 3:e65. 
16. Tabei $Y$, Tsuda K, Kin T, Asai K: SCARNA: fast and accurate structural alignment of RNA sequences by matching fixedlength stem fragments. Bioinformatics 2006, 22: I723-I729.

17. Kiryu H, Tabei Y, Kin T, Asai K: Murlet: a practical multiple alignment tool for structural RNA sequences. Bioinformatics 2007, 23: I 588-I598.

18. $\mathrm{Xu} \mathrm{X}, \mathrm{Ji} \mathrm{Y}$, Stormo G: RNA Sampler: a new sampling based algorithm for common RNA secondary structure prediction and structural alignment. Bioinformatics 2007, 23:|883-|89|.

19. Holmes I: Accelerated probabilistic inference of RNA structure evolution. BMC Bioinformatics 2005, 6:73.

20. Dowell R, Eddy S: Efficient pairwise RNA structure prediction and alignment using sequence alignment constraints. $B M C$ Bioinformatics 2006, 7:400.

21. Taneda A: Cofolga: a genetic algorithm for finding the common folding of two RNAs. Comput Biol Chem 2005, 29: I I I- I I9.

22. Torarinsson E, Sawera M, Havgaard J, Fredholm M, Gorodkin J: Thousands of corresponding human and mouse genomic regions unalignable in primary sequence contain common RNA structure. Genome Res 2006, 1 6:885-889.

23. Uzilov A, Keegan J, Mathews D: Detection of non-coding RNAs on the basis of predicted secondary structure formation free energy change. BMC Bioinformatics 2006, 7:I73.

24. Torarinsson E, Yao Z, Wiklund E, Bramsen J, Hansen C, Kjems J, Tommerup N, Ruzzo W, Gorodkin J: Comparative genomics beyond sequence-based alignments: RNA structures in the ENCODE regions. Genome Res 2008, I 8:242-25I

25. Hofacker I, Fontana W, Stadler P, Bonhoeffer L, Tacker M, Schuster P: Fast Folding and Comparison of RNA Secondary Structures. Monatsh Chem 1994, I25:167-188.

26. Notredame C, O'Brien E, Higgins D: RAGA: RNA sequence alignment by genetic algorithm. Nucleic Acids Res 1997, 25:4570-4580.

27. Goldberg DE: Genetic Algorithms in Search, Optimization and Machine learning New York: Addison-Wesley; 1987.

28. Needleman S, Wunsch C: A general method applicable to the search for similarities in the amino acid sequence of two proteins. I Mol Biol 1970, 48:443-453.

29. Notredame C, Higgins D: SAGA: sequence alignment by genetic algorithm. Nucleic Acids Res 1996, 24: I5 I5-I524.

30. Gerstein $M$, Levitt $M$ : Using iterative dynamic programming to obtain accurate pairwise and multiple alignments of protein structures. Proc Int Conf Intell Syst Mol Biol 1996, 4:59-67.

31. Dalli D, Wilm A, Mainz I, Steger G: STRAL: progressive alignment of non-coding RNA using base pairing probability vectors in quadratic time. Bioinformatics 2006, 22:1593-1599.

32. Klein R, Eddy S: RSEARCH: finding homologs of single structured RNA sequences. BMC Bioinformatics 2003, 4:44

33. Nussinov R, Pieczenik G, Griggs J, Kleitman D: Algorithm for loop matching. SIAM J Appl Math 1978, 35:68-82.

34. Hofacker I, Fekete M, Stadler P: Secondary structure prediction for aligned RNA sequences. I Mol Biol 2002, 3 I 9: I059-1 066.

35. Knudsen B, Hein J: Pfold: RNA secondary structure prediction using stochastic context-free grammars. Nucleic Acids Res 2003, 3 I:3423-3428

36. Thompson J, Plewniak F, Poch O: A benchmark alignments database for the evaluation of multiple sequence alignment programs. Bioinformatics 1999, 15:87-88.

37. Wilm A, Mainz I, Steger G: An enhanced RNA alignment benchmark for sequence alignment programs. Algorithms Mol Biol 2006, I:19.

38. Selig C, Wolf M, Müller T, Dandekar T, Schultz J: The ITS2 Database II: homology modelling RNA structure for molecular systematics. Nucleic Acids Res 2008 in press. http://nar.oxfordjour nals.org/cgi/content/abstract/gkm827vl. [10.1093/nar/gkm827]

39. Schultz J, Maisel S, Gerlach D, Müller T, Wolf M: A common core of secondary structure of the internal transcribed spacer 2 (ITS2) throughout the Eukaryota. RNA 2005, I I(436I-364 [http://www.rnajournal.org/cgi/content/abstract/II/4/36I].

40. Cofolga2 home page [http://rna.eit.hirosaki-u.ac.jp/cofolga2/new/ ]

41. Gorodkin J, Stricklin S, Stormo G: Discovering common stemloop motifs in unaligned RNA sequences. Nucleic Acids Res 200I, 29:2 I35-2| 44.

42. Chang CC, Lin CJ: LIBSVM: a library for support vector machines 200 [http://www.csie.ntu.edu.tw/ cilin/libsvm/].
43. Uzilov A, Keegan J, Mathews D: Detection of non-coding RNAs on the basis of predicted secondary structure formation free energy change. BMC Bioinformatics 2006, 7:173.

44. Cherry J, Ball C, Weng S, Juvik G, Schmidt R, Adler C, Dunn B, Dwight S, Riles L, Mortimer R, Botstein D: Genetic and physical maps of Saccharomyces cerevisiae. Nature 1997, 387:67-73.

45. Gish W: WU-BLAST home page. 1996 [http://blast.wustl.edu]

46. Torarinsson E, Sawera M, Havgaard J, Fredholm M, Gorodkin J: Thousands of corresponding human and mouse genomic regions unalignable in primary sequence contain common RNA structure. Genome Res 2006, 16:885-889.

47. Harmanci A, Sharma G, Mathews D: PARTS: Probabilistic Alignment for RNA joinT Secondary structure prediction. Nucleic Acids Res 2008, 36:2406-24I7.

48. Havgaard J, Torarinsson E, Gorodkin J: Fast Pairwise Structura RNA Alignments by Pruning of the Dynamical Programming Matrix. PLoS Comput Biol 2007, 3(10): I896-1908.

49. Washietl S, Hofacker I, Lukasser M, Huttenhofer A, Stadler P: Mapping of conserved RNA secondary structures predicts thousands of functional noncoding RNAs in the human genome. Nat Biotechnol 2005, 23: I383-1390.

50. David L, Huber W, Granovskaia M, Toedling J, Palm C, Bofkin L, Jones $\mathrm{T}$, Davis R, Steinmetz L: A high-resolution map of transcription in the yeast genome. Proc Natl Acad Sci USA 2006, 103:5320-5325.

51. Davis CMA Jr: Accumulation of unstable promoter-associated transcripts upon loss of the nuclear exosome subunit Rrp6p in Saccharomyces cerevisia. Proc Natl Acad Sci USA 2006, 1 03:3262-3267.

52. Samanta M, Tongprasit W, Sethi H, Chin C, Stolc V: Global identification of noncoding RNAs in Saccharomyces cerevisiae by modulating an essential RNA processing pathway. Proc Nat Acad Sci USA 2006, 103:4192-4197.

53. Miura F, Kawaguchi N, Sese J, Toyoda A, Hattori M, Morishita S, Ito $T$ : A large-scale full-length cDNA analysis to explore the budding yeast transcriptome. Proc Natl Acad Sci USA 2006, 103:17846-|785I.

54. Gonsalvez G, Urbinati CR, Long RM: RNA localization in yeast: moving towards a mechanism. Biol Cell 2005, 97:75-86.

55. Bauer M, Klau G, Reinert K: Accurate multiple sequence-structure alignment of RNA sequences using combinatorial optimization. BMC Bioinformatics 2007, 8:27I.

56. Dalli D, Wilm A, Mainz I, Steger G: STRAL: progressive alignment of non-coding RNA using base pairing probability vectors in quadratic time. Bioinformatics 2006, 22:1593-I599.

57. Katoh K, Misawa K, Kuma K, Miyata T: MAFFT: a novel method for rapid multiple sequence alignment based on fast Fourier transform. Nucleic Acids Res 2002, 30:3059-3066.

58. Thompson J, Higgins D, Gibson T: CLUSTAL W: improving the sensitivity of progressive multiple sequence alignment through sequence weighting, position-specific gap penalties and weight matrix choice. Nucleic Acids Res 1994, 22:4673-4680.

Publish with Biomed Central and every scientist can read your work free of charge

"BioMed Central will be the most significant development for disseminating the results of biomedical research in our lifetime. "

Sir Paul Nurse, Cancer Research UK

Your research papers will be:

- available free of charge to the entire biomedical community

- peer reviewed and published immediately upon acceptance

- cited in PubMed and archived on PubMed Centra

- yours - you keep the copyright 